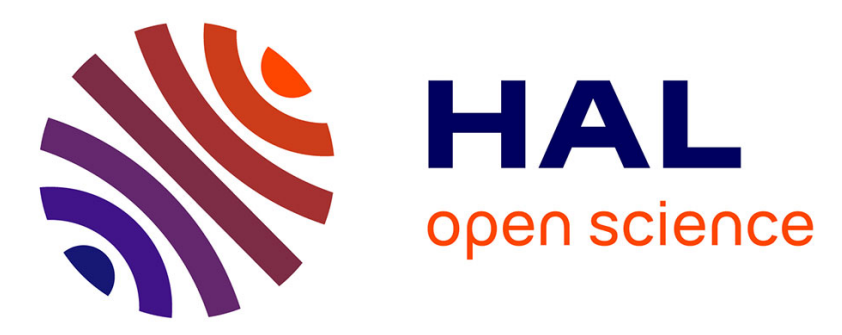

\title{
Influence of the energy dissipation rate in the discharge of a plasma synthetic jet actuator
}

Antoine Belinger, P Hardy, P Barricau, Jean-Pascal Cambronne, D Caruana

\section{To cite this version:}

Antoine Belinger, P Hardy, P Barricau, Jean-Pascal Cambronne, D Caruana. Influence of the energy dissipation rate in the discharge of a plasma synthetic jet actuator. Journal of Physics D: Applied Physics, 2011, 44 (36), pp.365201. 10.1088/0022-3727/44/36/365201 . hal-00651637

\section{HAL Id: hal-00651637 https://hal.science/hal-00651637}

Submitted on 14 Dec 2011

HAL is a multi-disciplinary open access archive for the deposit and dissemination of scientific research documents, whether they are published or not. The documents may come from teaching and research institutions in France or abroad, or from public or private research centers.
L'archive ouverte pluridisciplinaire HAL, est destinée au dépôt et à la diffusion de documents scientifiques de niveau recherche, publiés ou non, émanant des établissements d'enseignement et de recherche français ou étrangers, des laboratoires publics ou privés. 


\title{
Influence of the energy dissipation rate in the discharge of a Plasma Synthetic Jet Actuator
}

\author{
A Belinger ${ }^{1,2}$, P Hardy ${ }^{3}$, P Barricau ${ }^{3}, \mathrm{~J} \mathrm{P} \mathrm{Cambronne}^{1,2}$ and D Caruana ${ }^{3}$ \\ ${ }^{1}$ Université de Toulouse ; UPS, INPT ; LAPLACE (Laboratoire Plasma et Conversion \\ d'Energie) ; 118 route de Narbonne, F-31062 Toulouse cedex 9, France \\ ${ }^{2}$ CNRS ; LAPLACE ; F-31062 Toulouse, France. \\ ${ }^{3}$ ONERA Centre Midi-Pyrénées; Département Modèles pour l'Aérodynamique et \\ l'Energétique ; BP74025, 2 avenue Edouard Belin ; 31055 TOULOUSE CEDEX 4, \\ France
}

E-mail : daniel.caruana@onera.fr

\begin{abstract}
A promising actuator for high-speed flow control, referred to as a Plasma Synthetic Jet (P.S.J), is being studied by the DMAE department of the ONERA, and the Laplace laboratory of the CNRS, in France. This actuator was inspired by the "Sparkjet" device developed by the Johns Hopkins University Applied Physics Laboratory. The PSJ, which produces a synthetic jet with high exhaust velocities, no active mechanical components and no mass flow admission, holds the promise of enabling high-speed flows to be manipulated. With this high velocity jet it is possible to reduce fluid phenomena such as transition and turbulence, thus making it possible to increase an aircraft's performance whilst at the same time reducing its environmental impact. A thermal plasma discharge was created in a micro cavity, causing the gas to be expelled. It is relevant that the velocity and momentum depend on the energy dispersed by the electric discharge. To control the frequency and energy dispersed in the plasma, the Laplace laboratory has developed two high voltage power supply systems. These allow two different types of discharge to be produced, with energy being supplied to the discharge in two different manners. In this paper, we focus on the impact of the power supply on the Plasma Synthetic Jet, and in particular on the role of the rate of energy dissipation in the discharge. In order to estimate the influence of the power supply on the machinery of the actuator, specific experimental techniques were used to investigate the electrical (voltage, current), thermal (Infra-red camera) and aerodynamic (jet duration, isentropic pressure, jet velocity) characteristics. These data sets were used to determine which of the two power supplies was more effective, thus allowing us to reach several conclusions concerning the importance of the energy dissipation rate on the PSJ actuator.
\end{abstract}

1. Keywords

- Plasma actuator

- Synthetic Jet

- Spark discharge 
- Flow control

\section{Introduction}

The development of flow control applications with active devices requires efficient, robust, easy to integrate and low energy consumption actuators. In recent years, various studies have been conducted on electrostatic and electromagnetic actuators. Actuators making use of piezoelectric materials [1-3] or plasma [4-15] are also being developed. There are several possible configurations for piezoelectric actuators, including: a synthetic jet [1-2] and a flat plat actuator [3]. Wilste and Glezer have shown that a flat plate actuator is able to control a shear layer [3]. Smith and Glezer [1] presented modifications which can be achieved in the jet, by means of a piezoelectric actuator. Mane analyzed [2] the influence of the sawtooth, the orifice diameter, and the cavity height on the performance of the actuator, for three different diaphragm materials.

Various aspects of plasma actuators have been extensively studied. Moreau [5] provides a general overview of the subject. This paper describes different types of actuator: surface corona discharge and surface dielectric barrier discharge actuators, and several types of airflow control for different configurations (cylinder, flat plate ...). Surface corona actuators have shown the ability to control external flow in a flat plate [4], or in a cylinder [6]. In [7], Corke and Orlov provide a general review of the Surface Dielectric Barrier Discharge actuator, and of different applications such as boundary layer separation control. In [8], Boeuf and Pitchford calculated the electrodynamic force created by a DBD actuator. The authors concluded that SDBD and surface corona discharges create the same electrohydrodynamic effect. An original SDBS with multiple electrodes is presented in [9]. This actuator extends the plasma discharge and thus the control surface, when compared with a single SDBD. The SDBD actuator has also influenced new plasma actuator designs, for example a directional micro jet is presented in [10]. The velocity of all of these actuators is less than $10 \mathrm{~m} / \mathrm{s}$. A new plasma actuator referred to as the "sparkjet" has been proposed by the Johns Hopkins University Applied Physics Laboratory [11,12]. This actuator is able to produce high-speed jet velocities, with a peak velocity measured by the PIV technique in excess of $50 \mathrm{~m} / \mathrm{s}$ [13]. A pulsed plasma jet inspired by the sparkjet actuator is presented in [14], with a jet velocity of the order of $300 \mathrm{~m} / \mathrm{s}$. The ONERA, in 
collaboration with the CNRS Laplace laboratory (France), is also involved in research into a PSJ actuator [15]. The Laplace laboratory is more particularly interested in the interactions between the power supply and the PSJ actuator. Indeed, the impact of the power supply on the plasma can modify the processes which occur, for example, in dielectric barrier discharge lamps [16-18] or ozone generator [19-21]. In [16], Mildren showed that the efficiency and light intensity (between 175 and $200 \mathrm{~nm}$ ) are improved when the lamps are supplied by a pulsed power supply (rather than an AC power supply), and photographs of the light emission are presented in [17]. When using an ozone generator, Williamson obtained the same results [19], with the pulsed power supply producing more ozone than an AC power supply. Consequently, various researchers have investigated the role of the power supply for ozone generation. For example, in [21] Ordiz presents a buck converter, and in [20] Koudriavtsev et al. present a resonant power supply. The supply strategy influences the process and radically changes the efficiency and performance of the application. The influence of the power supply on the plasma has also been studied for flow control applications. Jolibois showed that the high voltage waveform changes the electric wind produced by SDBD [22]. Indeed, a negative sawtooth leads to a higher velocity than a positive sawtooth [23], since the former produces a high current peak, and thus a more intense filament, whereas a positive sawtooth produces a current peak of lower amplitude. The frequency of the high voltage modulation also influences the velocity of the electric wind produced by the SDBD [24].

The influence of the power supply on the plasma, and consequently on the process, have not yet been studied on the PSJ actuator. In the present paper, we propose to compare two power supplies, in particular the influence of the energy dissipation rate on the jet velocity. We thus compare the jet velocity, and the dynamic pressure produced by the PSJ actuator supplied by two different power supplies: an inductive power supply (IPS), and a capacitive power supply (CPS). The CPS produces a pulsed plasma, whose energy is rapidly dissipated by the discharge, whereas the IPS causes the discharge energy to dissipate more slowly. In the first section of this paper, the discharge created by each of these power supplies is described, and an electrical model of the discharges is presented. In the second section, the dissipation rate of the discharge energy is analyzed for both power supplies. Finally, the velocity, gas expulsion time and total pressure of both power supplies are compared. 


\section{Experimental set-up}

\subsection{Plasma synthetic Jet}

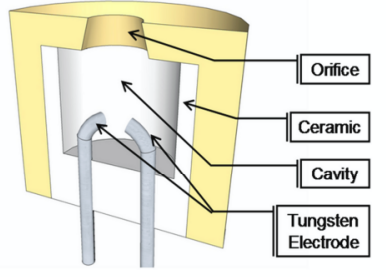

( a )

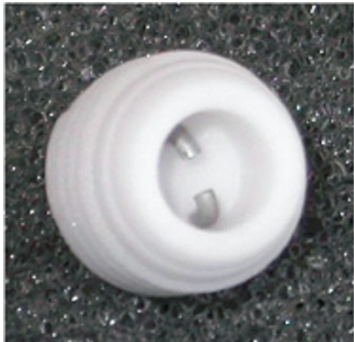

( b )

Figure 1. Plasma Synthetic Jet actuator : (a) sectional view; and (b) view of the actuator without the cap

A PSJ comprises three main elements: a pair of tungsten electrodes separated by a $1.2 \mathrm{~mm}$ air gap, a brass cap, and a ceramic cavity. The cavity of the PSJ used in this work has a volume of $50 \mathrm{~mm}^{3}$, indeed the diameter and the height of the cavity are both equal to $4 \mathrm{~mm}$. As shown in Figure 1, the two electrodes are enclosed in the ceramic, and an orifice is positioned at the top of the cavity. At the beginning of the cycle, a high voltage is applied between the two electrodes, by means of the high voltage power supply described in the following subsection. When the voltage reaches the disruptive voltage of air (approximately $4700 \mathrm{~V}$ for this actuator), air is ionized and an electric arc is created. This discharge leads to a strong increase in the internal energy. Because the air is confined, its pressure and temperature increase rapidly. Relaxation of the fluid through the circular orifice creates a hot air jet. When temperature in cavity drops, ambient air is then sucked up into the cavity (recovery stage during some hundred of microsecond), and the actuator is ready for a new cycle. 


\subsection{Power supply}

In order to create the discharge, a high voltage power supply is essential. Moreover, this power supply must control the frequency of, and the energy dissipated in, the discharge. Two solutions can be used to create a high voltage:

- A high voltage power supply, associated with an IGBT switch, to commute the high voltage directly during the discharge,

- A low voltage power supply, associated with a high voltage transformer and a transistor.

The first solution would lead to very high costs, since it requires the use of a high voltage transistor. We thus chose the second approach, with a flyback converter [25]. In association with the flyback converter architecture, we proposed two different types of power supply. In the first of these, a PSJ is directly connected to the secondary voltage of the transformer; this solution is referred to as IPS it is inspired to the spark engine power supply [26]. The second solution is referred to as a CPS [27], and involves the use of a capacitor, inserted between the transformer and the PSJ. The supply mode is totally different for these two power supplies. In the first, the inductance transfers the energy to the discharge, such that the PSJ is powered by a current source. In the second, the capacitor transfers the energy to the discharge, such that the PSJ is powered by a voltage source. The functioning of these two power supplies is analyzed in the following section.

\subsection{Measurements}

3.3.1. Electrical Measurement. The actuator was supplied by a high voltage power supply, as described in the following section. The high voltage was measured by a $20 \mathrm{MHz}$ high voltage probe, and the current probe measured the AC current only, with a pass-band limited to $100 \mathrm{MHz}$. The data was recorded with a $100 \mathrm{MHz}$ LECROY oscilloscope. 


\subsubsection{Stagnation Pressure Measurements. For flow control applications (especially separation), the}

jet diameter of the active vortex generator is close to $1 \mathrm{~mm}$. Since it is difficult to achieve direct measurements of the PSJ characteristics, we used a dedicated instrumentation setup mounted on a calibration bench, thus enabling the dynamic pressure and velocity of the jet to be measured: this consisted in a stagnation pressure probe, with a differential unsteady KULITE \pm 5 psi sensor. A MACOR tube was fitted to the sensor. This device allows the jet to be channeled towards the sensitive element of the sensor. The probe was mounted on a micrometric translation plate, to ensure its accurate positioning in front of the actuator orifice.

3.3.3. Velocity Measurement. Velocity measurements are performed by means of the Schlieren technic. Since the jet is produced by a thermal source (electric arc), the resulting density (hence refractive index) gradients allow the position of the jet to be easily measured.

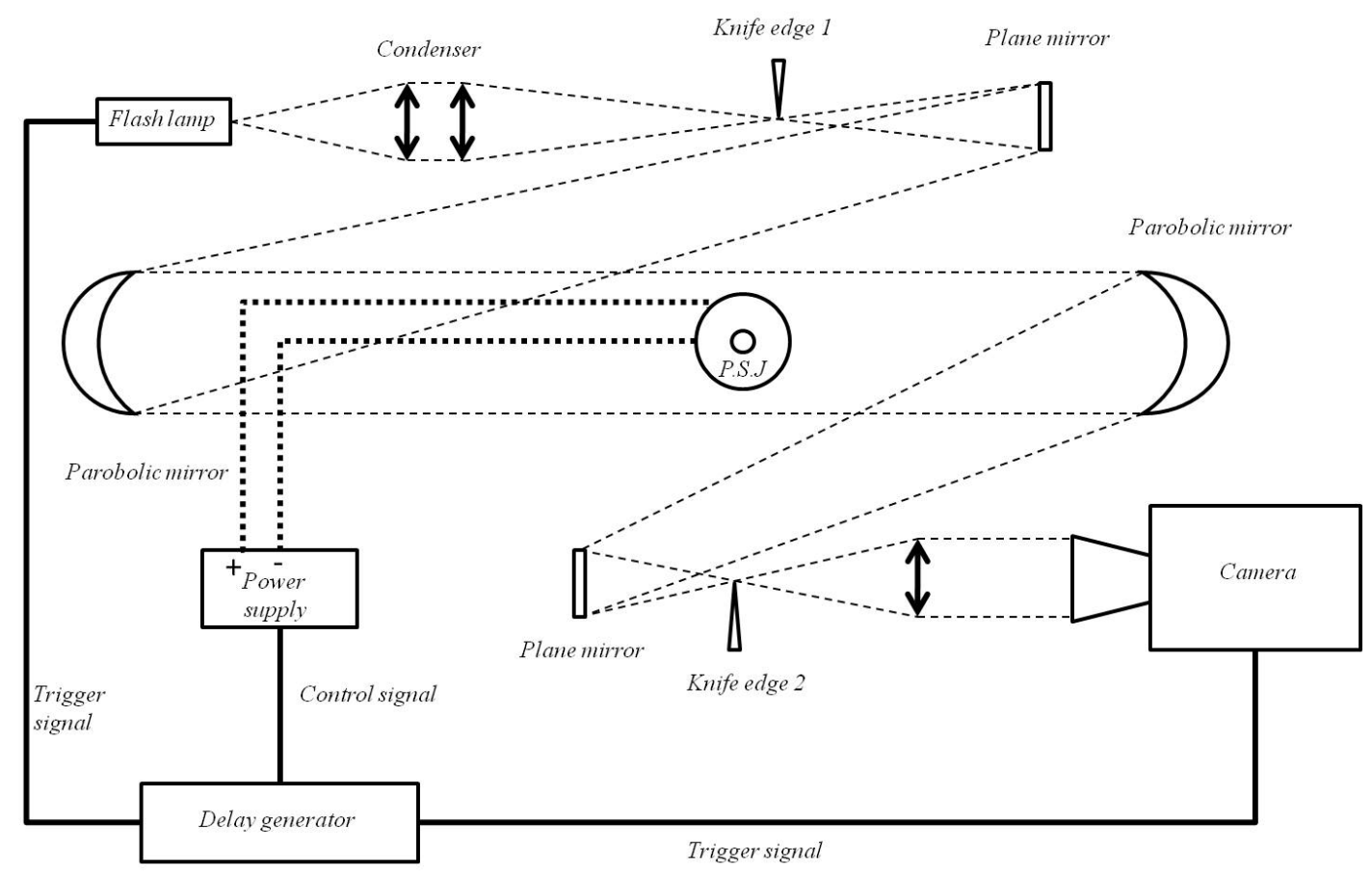

Figure 2. Schlieren setup

The Schlieren setup used a conventional Z-type mirror system as seen on Figure 2. The knife-edge was oriented orthogonally to the jet axis. This allowed the density gradient to be visualized in the streamwise direction. The actuator was mounted on an electrically isolated flat plane, parallel to the optical axis of the schlieren bench. Image frames were acquired by means of a MotionPro X5 camera. 
The camera CCD had a 2352 x 1728 pixel imaging matrix with a spatial resolution of $64 \mu \mathrm{m}$. For the purposes of the very short integration times needed for each frame, the illumination was produced by the flash from a Flash lamp (HSPS FX-Xenon), with typical durations of approximately $20 \mathrm{~ns}$.

The control signal of the power supply was used to synchronize the spark lamp with the camera acquisitions. A Standford Research Systems DG535 delay generator was triggered by the signal from the probe, allowing it to generate the TTL signals for the spark lamp and the camera. The position of the jet was thus a function of the phase difference between the spark of the PSJ and the frame acquisition. The jet's velocity could then be computed from the ratio between the distance it covered in the images, and the corresponding time delay. The jet's position was determined from the average value of several acquisitions.

The time difference used to determine the exhaust velocity varied between 5 and $10 \mu$ s, depending on which power supply was used. The maximum working frequency of the spark lamp limits the velocity measurement bench. The measurements are made with an actuator frequency of $10 \mathrm{~Hz}$.

\subsubsection{Temperature measurement}

It is too interesting to know the temperature of the gas expel by the PSJ actuator. However, the jet duration is too short to measure his temperature. That is the reason why we have measured the temperature of the top of the cap of the actuator with an infrared camera ThermoVision SC 6000 FLIR. This measurement allows us to quantify the temperature in the cavity before the breakdown. So we can know which power supply heats over the actuator.

An image of the cap is taken at a time interval of $5 \mathrm{~s}$. At each image, the cap temperature is uniform throughout the cap; it is certainly due to the small time of the device. As the emissivity of the brass is low, the infrared radiation is strongly attenuated. To have a better precision in this measurement, we recover the cap with high emissivity paint $(\varepsilon=0.92)$. To estimate the temperature we use the Plank law.

\section{Power supply}




\subsection{Energy transfer}

4.1.1. Magnetization stage. Figure 3 shows the circuit diagrams of the two power supplies. Both power supplies consist of a high-voltage transformer, an IGBT, a DC power supply (60 V - 500W) and a capacitor for the second power supply. The magnetizing inductance has a value of $10 \mathrm{mH}$, and the transformer's rated voltage ratio is equal to 10 .

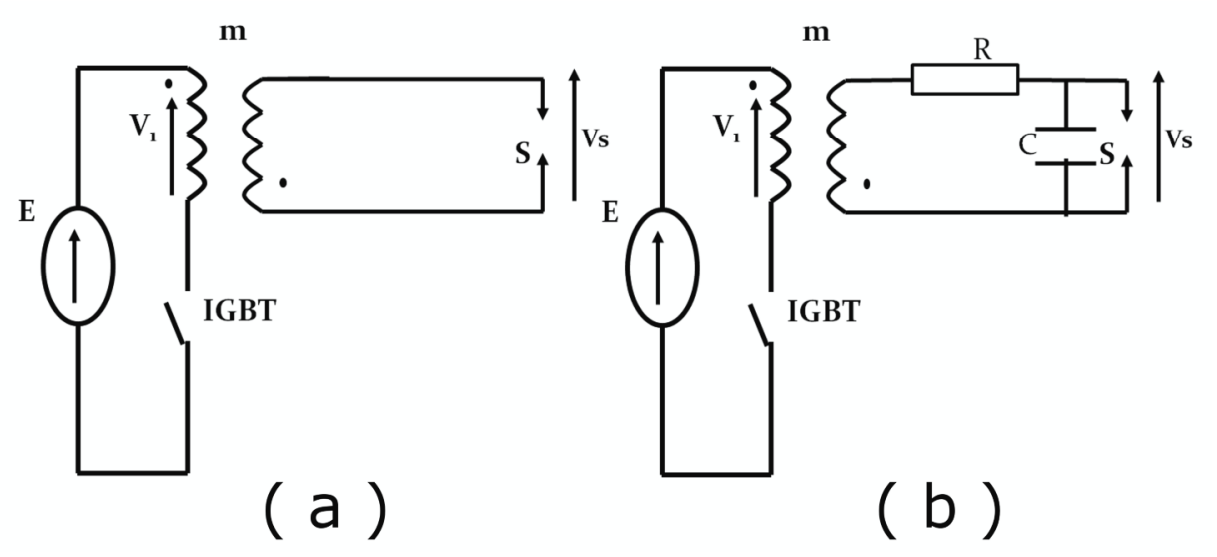

Figure 3. Inductive (a) and capacitive (b) power supplies

The magnetization stage is identical for both power supplies. The high-voltage transformer is powered by the low-voltage power supply during the on-state of the IGBT transistor. During this phase, the primary current increases linearly and energy is accumulated in the transformer. This energy is determined by the duration of the magnetization and depends on the square of the primary current $\left(\mathrm{I}_{\mathrm{p}}\right)$

$$
E_{m}=\frac{1}{2} \cdot L_{1} \cdot I_{p}^{2}
$$

where $\mathrm{L}_{1}$ represents the magnetizing inductance of the transformer. Moreover, the working frequency of the actuator is imposed by the IGBT frequency control. The IGBT can be easily controlled to adjust the frequency and the energy accumulated in the transformer. 
4.1.2. Energy transfer to the higher voltage. The magnetization phase ends with the opening of the IGBT transistor. The energy accumulated in the transformer is transferred to the secondary coil and thus to the discharge. The energy dissipated in the discharge $\left(\mathrm{E}_{\mathrm{d}}\right)$ can be calculated from the measurement of the discharge voltage (Vs) and the discharge current (Id):

$$
E=\int_{t_{\text {discharge }}} v_{s}(t) \cdot i_{d}(t) \cdot d t-R_{\text {wire }} \cdot \int_{t_{\text {discharge }}} i_{d}(t)^{2} \cdot d t
$$

Where $R_{\text {wire }}$ represents the resistance of the wire: $0.5 \Omega$ in this work.

\subsection{Inductive Power supply}

The magnetization phase ends with the opening of the IGBT. After switch-off of the IGBT, the secondary voltage increases until the breakdown voltage is reached. Figure 4 shows the ignition of the discharge. The current increases to $30 \mathrm{~A}$ in $50 \mathrm{~ns}$. During the ignition of the discharge, the voltage decreases rapidly to $400 \mathrm{~V}$ (this voltage is the same in every case).

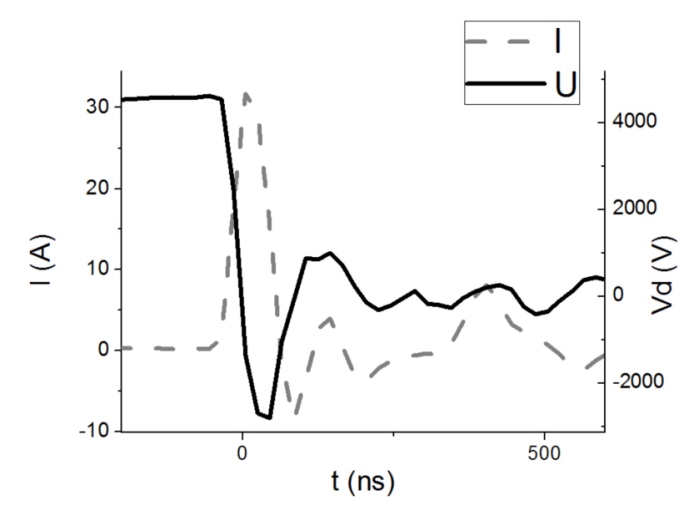

Figure 4. Ignition of the IPS discharge

Whatever the energy dissipated in the discharge, the ignition of the discharge is the same, and the current rapidly increases to $30 \mathrm{~A}$. This peak current value depends on the equivalent capacitance of the transformer wire, as described in section 3.3.2.

Following ignition, the duration and the maximum value of the discharge current varies with the energy accumulated in the transformer, as seen in Figure 5. 


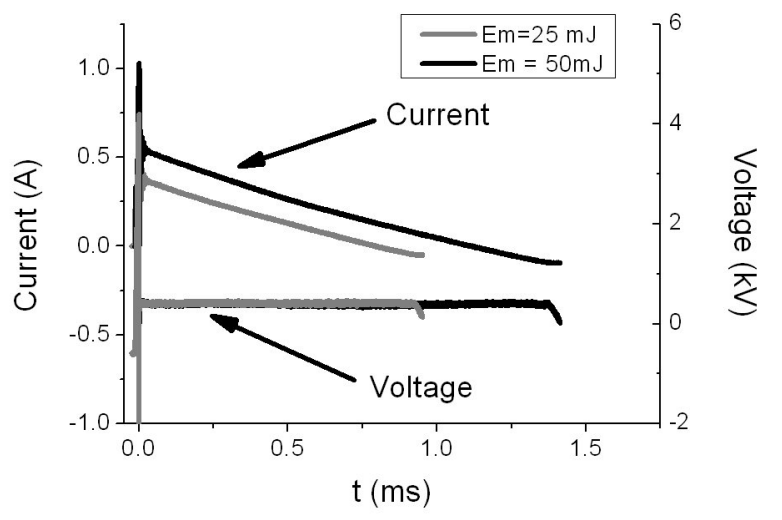

Figure 5. Discharge of the IPS

The discharge voltage and the slope of the decreasing current are constant, whatever the energy dissipated in the discharge. The slope of the current falloff is equal to:

$$
\frac{d i}{d t}=-\frac{U_{\text {disch } \arg e}}{L_{\mu_{-} \sec \text { ondary }}}
$$

and the constant voltage of the discharge is equal at: $\mathrm{U}_{\text {discharge }}=400 \mathrm{~V}$ As seen in [28-30], the glow discharge has the same electric characteristics, a constant voltage, and a variable current. Takaki [29] observed the same discharge voltage $(400 \mathrm{~V})$, and the current varied between 1 and a few milliamperes.

Moreover, the current and voltage waveforms are similar to those of the discharge of an inductance in series with a constant voltage source: since the current decreases linearly, it is controlled by the inductance and as a consequence the discharge behaves like a constant source voltage.

\subsection{Capacitive power supply}

In the CPS, the magnetization stage is terminated by the opening of the IGBT transistor. In an initial phase, the energy accumulated in the transformer, then transferred to the capacitor, is greater than that transferred to the discharge. The remainder of the energy is dissipated into a series resistance $(500 \Omega)$. 
If this resistance is disconnected, the terminal voltage of the transformer is non-zero when the IGBT is closed. A considerable increase in the IGBT current is thus induced by the energy transfer, which remains in the transformer. Unfotunately this resistance is required to protect the IGBT transistor. If the discharge is not initiated, the energy accumulated in the transformer is dissipated into the resistance. When the capacitor voltage on the actuator terminals reaches the electrical breakdown voltage (about $4700 \mathrm{~V}$ ), the capacitor delivers its energy through the discharge. The strong scattering of the breakdown voltage value (about $\pm 500 \mathrm{~V}$ ) imposes the storage of a greater quantity of energy in the transformer than in the capacitor. This large scattering in the breakdown voltage is certainly caused by the erosion of the electrode. Indeed this phenomenon can create some point in the electrode. This point increase locally the electric field and then diminish the breakdown voltage.

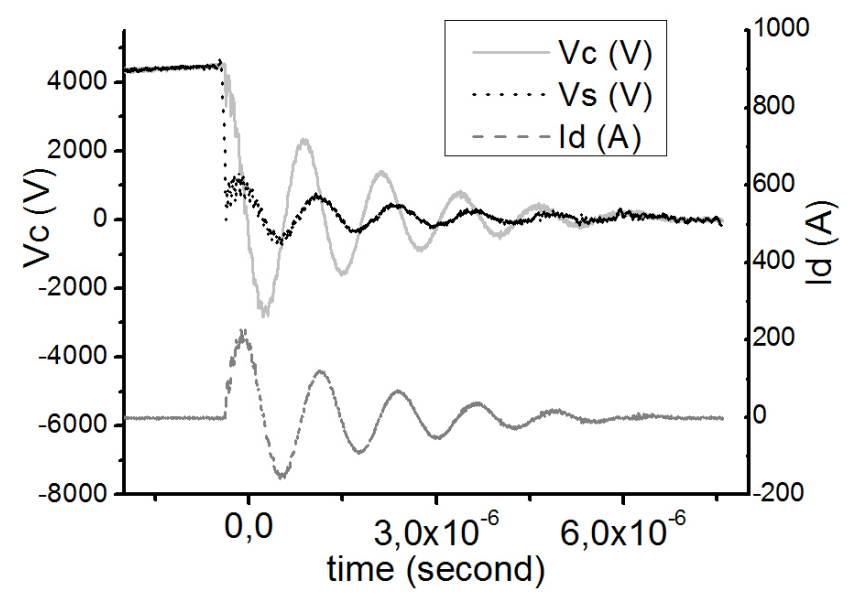

Figure 6. Discharge created by the CPS

Figure 6 provides a recording of the capacitor voltage and the discharge current during the gas conduction. The capacitor, the wire and the discharge behave like an oscillating circuit. The resistance and the inductance of the wire are measured by means of a network analyzer (passband $40 \mathrm{MHz}$ ). These parameters allow the discharge voltage to be calculated (Vs in Figure 6). Vs correspond at the real voltage between the electrode and $\mathrm{Vc}$ to the capacitor voltage. The difference between these two voltages corresponds to the wire voltage. Moreover, the wire is strongly inductive; consequently Vs and $\mathrm{Vc}$ are not in phase. During the ignition time, the discharge voltage falls to zero in a few nanoseconds. Following ignition, the current and the voltage discharge are sinusoidal and in phase. Furthermore, the maximum current is proportional to the breakdown voltage. In the same manner, the 
maximum value of the current increases as a function of the energy stored in the capacitor. Equation (4) shows the relation between capacitance, wire inductance and breakdown voltage.

$$
I_{s \max } \approx \sqrt{\frac{L_{\text {wire }}}{C}} \cdot V_{b}
$$

For a mean value of disruptive voltage $(4700 \mathrm{~V})$, the maximum current is plotted as a function of capacitance, as shown in Figure 7.

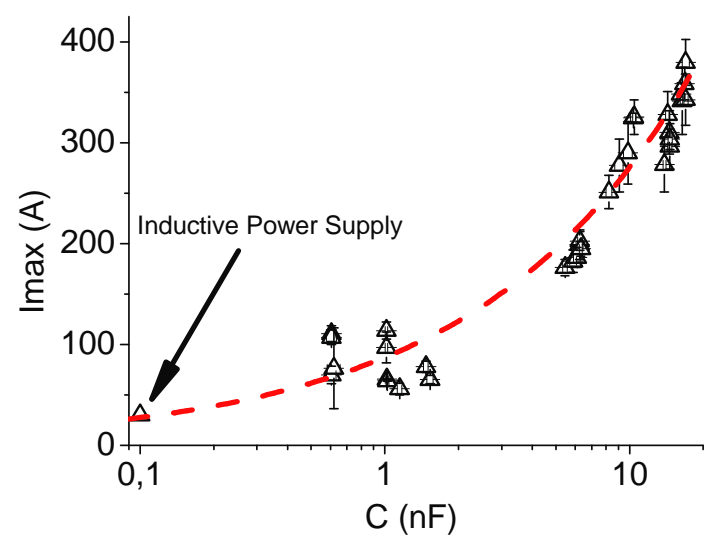

Figure 7. Maximal current as a function of capacitor value

The experimental points are in good agreement with the theoretical curve (the dotted line), the dispersion seen in Figure 7 being the consequence of variations in the breakdown voltage. The first experimental point represents the peak current of the IPS. Indeed, the capacitance of the wire in the transformer is equal to $100 \mathrm{pF}$, which explains the peak current of $30 \mathrm{~A}$. With both power supplies, the maximal current of the ignition of the discharge is influenced by the capacitor, such that the ignition mechanism is identical in both cases. Although the ignition is in principle only initiated by the capacitor, if the stored energy of the latter is very high, it may completely control the discharge.

The temporal variation of the current and the voltage indicates that the discharge behaves like a spark discharge [31-36]. 


\section{Influence of each power supply on the discharge behavior}

\subsection{Discharge Duration}

We measured the discharge duration between two instants in time:

- Ignition of the discharge, marked by the instant at which voltage breakdown occurs

- Termination of the discharge, marked by the current's fall to zero

The two power supplies applied the same energy to the discharge. Nevertheless, the energy was not dissipated in the same manner.

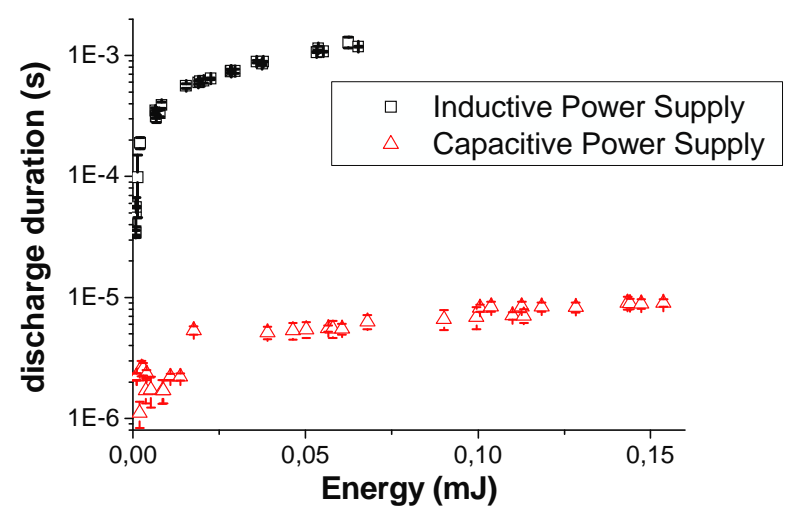

Figure 8. Discharge duration as a function of the energy dissipated during the discharge

Figure 8 plots the discharge measured duration, as a function of discharge energy, for the two power supplies. In the case of the IPS, this is not affected by the frequency. However, the discharge duration increases when the energy accumulated by the transformer is increased. This phenomenon leads to an increase in the magnetization time. On the one hand, the magnetization time increases the energy accumulated in the transformer and, consequently, the energy dissipated during the discharge. On the other hand, the discharge behaves like a constant voltage source, whatever the energy dissipated in the discharge. The maximum current thus increases with increasing energy. Since the discharge current decreases linearly, the discharge duration increases with the energy dissipated in the discharge. For the CPS, the discharge duration is also increased by the energy dissipated in the plasma. After $40 \mathrm{~mJ}$, the discharge duration is only weakly increased by the arc energy. Indeed, the arc duration is limited to 8 $\mu \mathrm{s}$. The IPS produces a more extended discharge than the CPS. 


\subsection{Energy dissipation}

Figure 9 presents the time evolution of the discharge energy dissipation, for a total discharge energy of $55 \mathrm{~mJ}$. This energy is dissipated more slowly by the IPS than by the CPS. With the CPS, we observe that the energy dissipation oscillates in time. Indeed, the energy is calculated from the capacitor voltage and the current of the discharge. Half of the total energy is dissipated during the ignition, in a period of 500 nano-seconds. The rest of the energy is then dissipated over a period of some microseconds.

In the case of the IPS, the energy is dissipated gradually, and ignition of the IPS consumes a small quantity of energy. Indeed, the maximum current is lower than in the CPS.

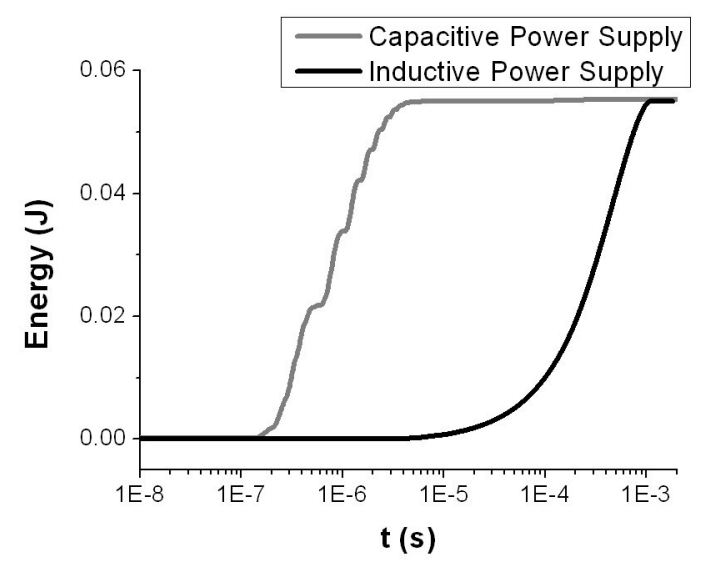

Figure 9. Temporal evolution of the energy dissipation

The stored energy is thus not transmitted in the same manner by the two power supplies. Consequently, the gas is not heated by the plasma at the same rate: heating of the gas is obtained more rapidly with the CPS. It is thus likely that the jet velocity and pressure are greater with this power supply. Indeed, the heating is more rapid, since the energy is dissipated in a shorter time. For the high energy of the IPS, the discharge lasts for 1 millisecond, such that gas expulsion begins before the electric discharge has finished. In this case, the heating effect is slower, and the resulting overpressure is certainly lower than in the case of the CPS. 


\subsection{Influence of the energy on the temperature of the cap}

The air in the cavity is not heated in the same way by the two power supply. Before the expulsion, air presents on the cavity heat the ceramic and the brass cap. Consequently, the temperature of the cap is representative of the heating of the air present in the cavity by the discharge. Figure 10 present the evolution of the cap temperature with the energy for the both power supply, frequency of the discharge is equal to $100 \mathrm{~Hz}$. The cap's temperature is stabilized after few min of functioning, so we plot this temperature versus energy dissipated in the discharge.

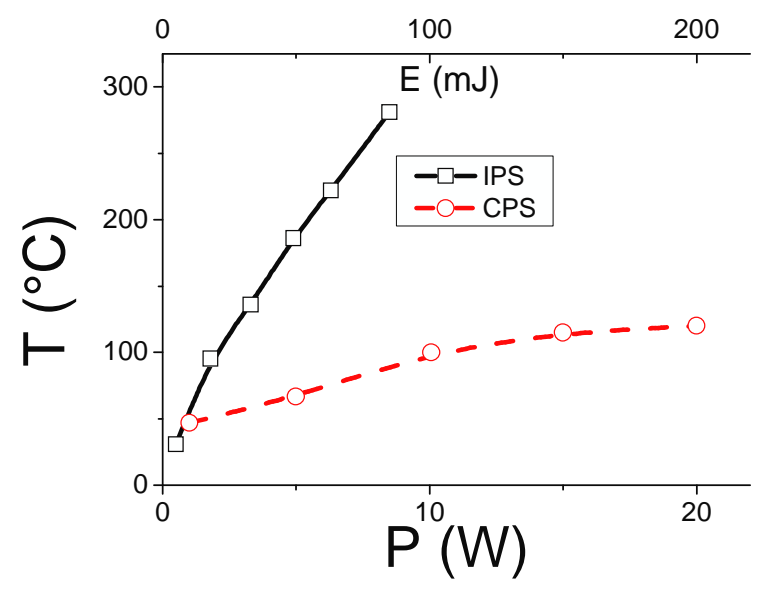

\section{Figure 10. Evolution of the cap temperature with the energy dissipated on the discharge}

It appears clearly than discharge created by the ISP heat hardly the actuator, consequently it is impossible to operate the actuator with this power supply at high frequency or at high energy dissipated in the discharge. Indeed, the cap's temperature depends directly of the power, which is directly linked to the frequency and to the energy dissipated in the discharge. The temperature of the cap is less hot with the CPS, but still reached $100^{\circ} \mathrm{C}$ for a power of $20 \mathrm{~W}$.

The energy dissipation rate influences strongly the temperature of the cap and therefore the heating of the gas present of the cavity before the breakdown voltage. With the IPS power supply the gas present in the cavity is inevitably warmer than for the CPS before the discharge. 


\subsection{Effect of the rate of energy dissipation on the breakdown voltage}

According to Paschen's theory, the breakdown voltage depends on the product of the electrode gap and the pressure, but also on the temperature. In our actuator, the breakdown voltage depends only on the temperature and on the pressure; indeed the electrode gap is constant. In order to show this assumption, we measured the breakdown voltage for different value of pressure or temperature inside the PSJ actuator. To change these parameters, the actuator without this cap is placed in a climatic chamber. In this chamber, the pressure can vary between 0.1 and 1 bar and the temperature between 20 ${ }^{\circ} \mathrm{C}$ and $180^{\circ} \mathrm{C}$. The cap of the actuator is removed in order to control the temperature and the pressure inside the cavity. In one experiment, the pressure is maintained to the atmospheric pressure and we change the temperature inside the climatic chamber. In another experiment the temperature is maintained at ambient temperature and we change the pressure in the climatic chamber. The evolution of the breakdown for this both experiment is plotted in function of the ratio temperature over pressure in Figure 11. This ratio corresponds to an equivalent temperature at atmospheric pressure and corresponds to the evolution of the gas density.

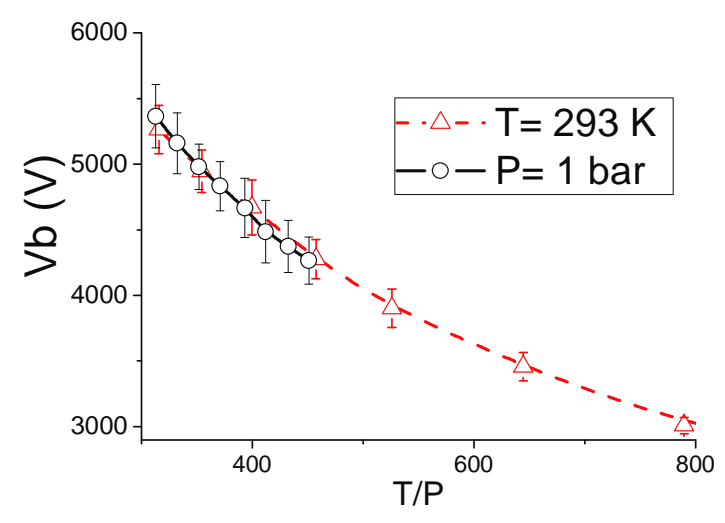

Figure 11: Evolution of the breakdown voltage with an equivalent temperature

The evolution of $V_{b}$ is the same for these two experiments because the two curves are stacked up. Consequently, these two parameters have the same influence in the breakdown voltage. Figure 12 present the evolution of the breakdown voltage for the two power supplies with the frequency. The measurement is carried out few minutes after the beginning of the actuator functioning. 


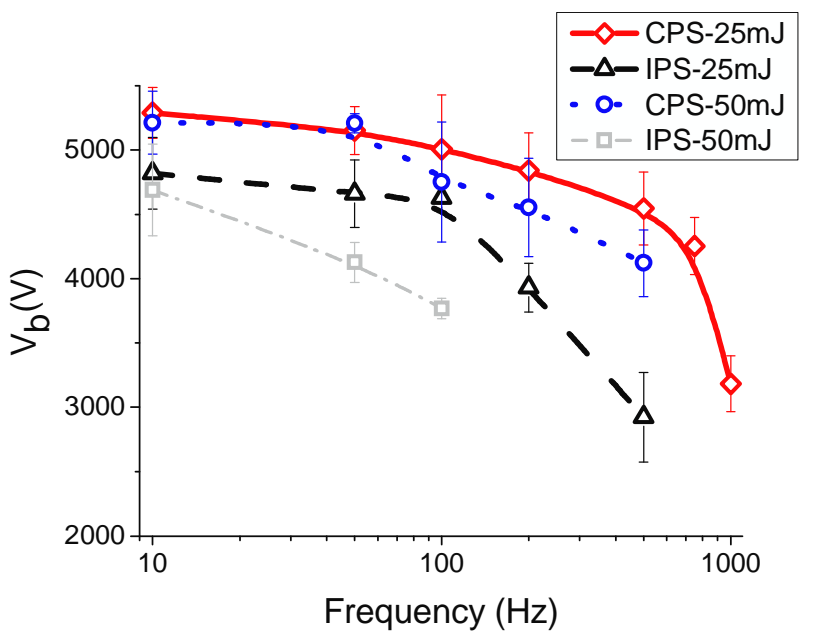

Figure 12. Breakdown voltage of the two power supplies

We want now to know if the drop of $\mathrm{V}_{\mathrm{b}}$ is due to the pressure or temperature. So we show the evolution of the disruptive voltage and of the cap temperature during the beginning of the functioning of the PSJ, indeed the thermal effect is negligible during this phase. The cap temperature measured with the infra-red camera is equal to the ambient temperature. So the gas temperature before the discharge is equal to the ambient air, because the cavity wall heat the air sucked during the recovery stage. Moreover the breakdown voltage remains unchanged for the first breakdown which corresponds to the beginning of the functioning of the PSJ. Consequently, at the end of the recovery stage, the pressure in the chamber is equal to the atmospheric pressure. Then, the drop of the breakdown voltage with the frequency and with the energy dissipated in the cavity is caused by the increase of the temperature in the cavity before the breakdown. Indeed if the temperature increases, the density of the gas decreases together with the breakdown voltage.

On the one hand, at the same frequency and for the same equivalent energy, the breakdown voltage of the IPS is smaller than the breakdown voltage of the CPS. On the other hand, the decrease in breakdown voltage as a function of accumulated energy is more significant for the IPS. The breakdown voltage for the two power supplies decreases with the frequency of the actuator, and with the energy accumulated by the transformer or the capacitor, as shown in Figure 12.

Consequently, the IPS heats the cavity more strongly than the CPS. This phenomenon can be understood from the energy dissipation rate and from the temperature cap. Indeed, with the IPS, the 
energy is dissipated over a longer period of time and the cap temperature is higher. The gas present in the cavity is thus ejected before the electric discharge has ended as shown in Figure 13.

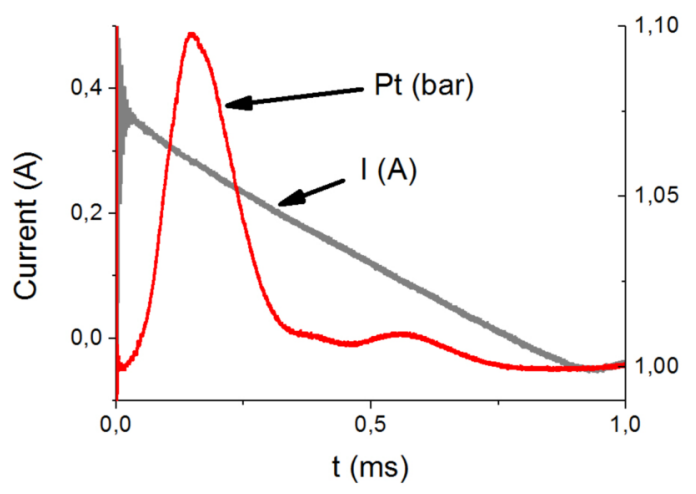

Figure 13: Evolution of the current with the pressure signal during the gas expulsion

The remaining energy heats only a small quantity of gas, such that most of this heat is dissipated in the ceramic of the actuator, which becomes hotter, before reaching the recovery stage. In summary, the air sucked into the cavity is hotter with the IPS. For the CPS, the discharge is too short (some microseconds) for the gas to be ejected before the discharge has ended.

For these reasons the breakdown voltage of the IPS is lower than that of the CPS.

\section{Influence of power supplies on the jet characteristics}

The dynamic pressure is measured by a pressure sensor tube, which is located a few millimeters in front of the PSJ orifice.

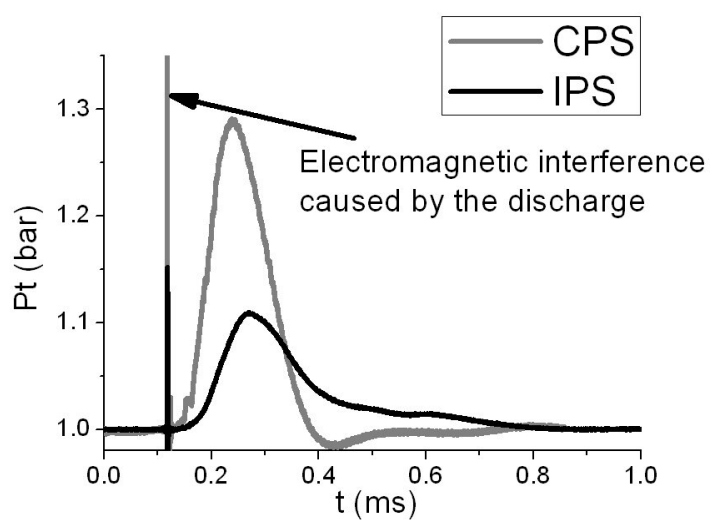

Figure 14. Signal of the dynamic pressure sensor 
With the CPS, for which the typically bell-shaped signal is shown in Figure 14, the jet is developed some micro seconds after the discharge. After the bell, the pressure signal is lower than 1 bar, this phase correspond to a violent suction of air by the PSJ actuator. This depression affects the pressure sensor and sucked the air present in the MACOR tube into the cavity. Indeed the expulsion phase ends very abruptly. This perturbation is not present in the signal corresponding to the IPS because the expulsion is more gradual and ends slowly, so the transition from the expulsion to the recovery stage is more progressive.

With the IPS, the pressure signal does not have exactly the same shape because the discharge continues during the gas expulsion. For the both power supply we can see the electromagnetic interference due to the discharge.

\subsection{Maximum dynamic pressure}

The maximum dynamic pressure, as a function of frequency, for different energies accumulated in the transformer or in the capacitor, is shown in Figure 15.

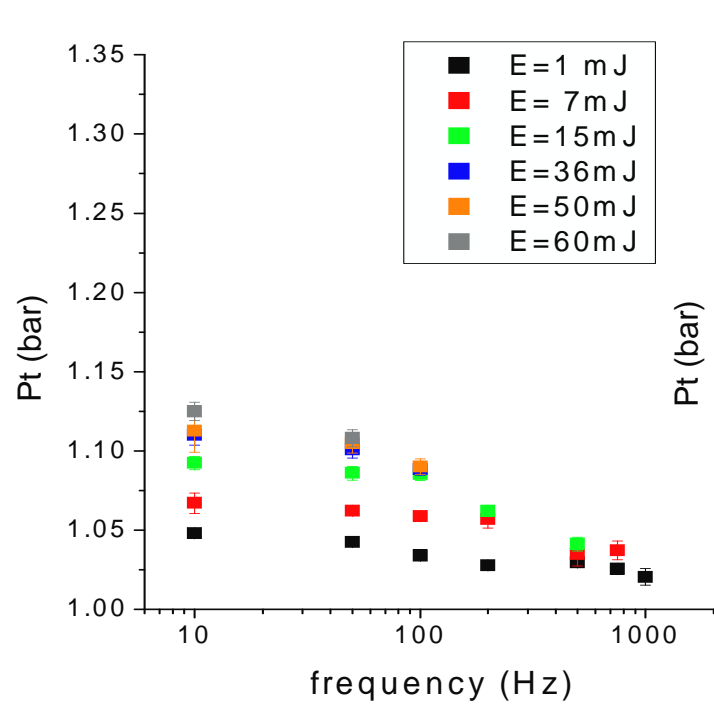

( a )

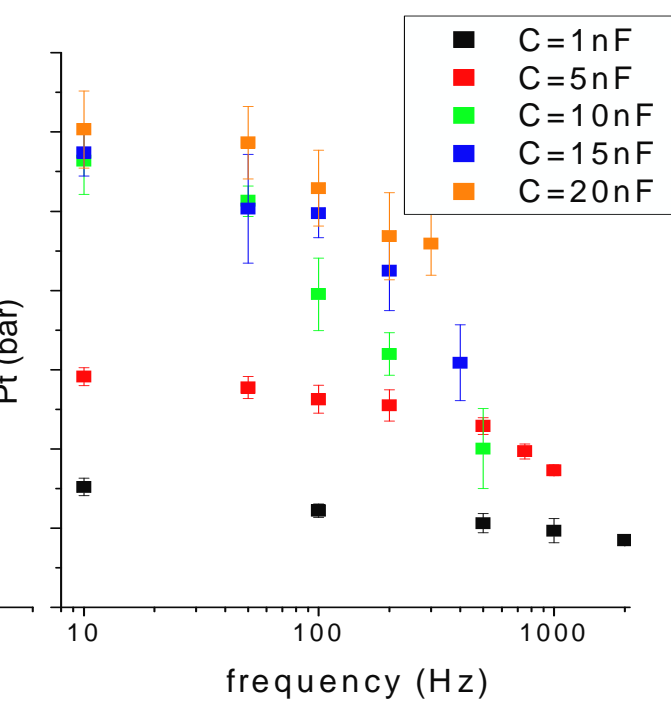

(b)

Figure 15. Maximum Total pressure of the two power supplies: ( a ) inductive power supply; and ( b ) capacitive power supply

The CPS produces a more powerful jet than the IPS. This can be explained by the brevity of the CPS discharge, and by the fact that it is not complete when the gas starts to be expelled by the IPS (Figure 
13): during expulsion of the gas, the heating is less efficient, because the gas's concentration in the cavity is low. This has the effect of heating the ceramic cavity which will warm the air during the suction. We have seen this effect in the cap temperature in Figure 10. Before the breakdown, the gas temperature in the cavity is then lower for the ISP than the CPS. This temperature is directly linked to the quantity of matter inside the cavity. The gas density before breakdown is then lower for the IPS than the CPS. Moreover, lower the density is, lower the quantity of gas that can be expelled by the PSJ is. The resulting pressure is then reduced for the IPS.

For the both power supplies, the dynamic pressure decreases at higher energies and higher frequency and then when the power dissipated in the PSJ is important. This drop is caused by the heating of the cavity. In the case of the CPS, the heating of the cavity is not the only reason for the fall in total pressure. Indeed, the breakdown voltage, and thus the energy dissipated during the discharge, is reduced by the heating of the cavity because of the relationship between capacitor energy and breakdown voltage.

$$
E_{c} \approx \frac{1}{2} \cdot C \cdot V_{b}^{2}
$$

The drop-off in total pressure is thus also due to the decline in energy dissipated during the discharge. The CPS power supply is somehow self regulated because the heating of the cavity induces a fall in the breakdown voltage which decreases the energy dissipated during the discharge. The temperature in the cavity is then lower which induces a diminution of the heating of the cavity.

\subsection{Gas expulsion time}

The gas expulsion time is estimated as the period during which the total pressure is greater than ten percent of the maximum over pressure. The over pressure correspond to pressure measured by the sensor which we subtract the atmospheric pressure.

Figure 16 shows the gas expulsion time as a function of the energy dissipated in the discharge. For the CPS, this time is constant $(220 \mu \mathrm{s})$, whatever the energy dissipated in the discharge. For the IPS, the 
gas expulsion time increases with energy (until $25 \mathrm{~mJ}$ ). Beyond $25 \mathrm{~mJ}$, the gas expulsion time is almost constant, and equal at $800 \mu \mathrm{s}$.

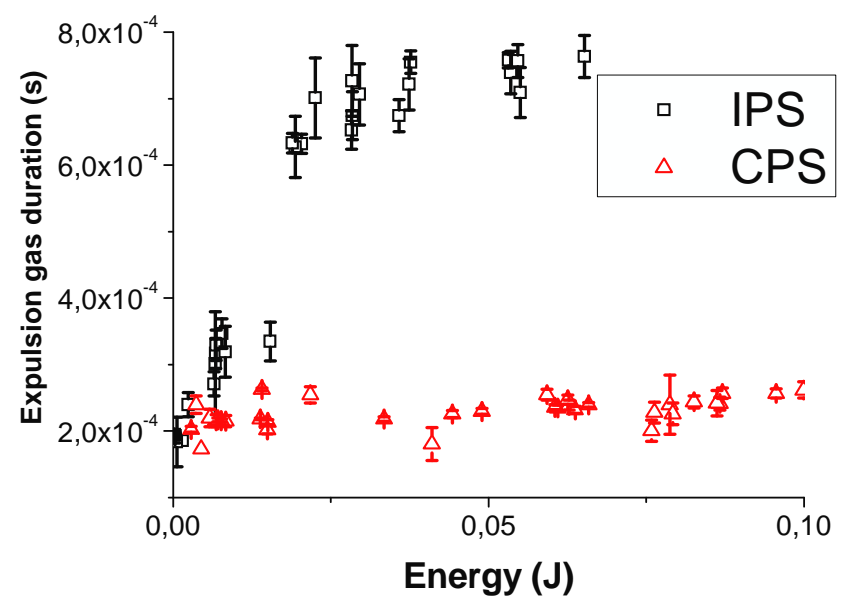

Figure 16. Gas expulsion time

The jet created by the IPS is expelled from the PSJ actuator during a relatively long time interval, whereas the CPS produces a short, pulsed jet.

Indeed, in the latter case, the gas expulsion time remains constant as a function of the energy or frequency. Consequently, if the discharge is too short, the discharge time will simply influence the maximum total pressure, without affecting the gas expulsion time.

However, in the case of the IPS, the gas expulsion time is influenced by the discharge time. Indeed, the jet is maintained because the IPS continues to discharge energy during expulsion of the gas. With the IPS at high energies, the discharge time is longer than the gas expulsion time, such that when the jet has been completely expelled, the discharge has still not come to an end. This phenomenon explains the stagnation of the gas expulsion time at higher energies.

\subsection{Velocity calculation}

The jet velocity was also calculated, on the basis of the total pressure measurements for frequency equal to $10 \mathrm{~Hz}$. If the jet flow is considered to be isentropic: 


$$
\frac{P_{t}}{P_{a}}=\left(1+\frac{\gamma-1}{2} M^{2}\right)^{\frac{1}{\gamma-1}}
$$

where

$$
M=\frac{V}{\sqrt{\gamma \cdot R \cdot T}}
$$

and $\mathrm{T}$ is the temperature of the jet, $\mathrm{M}$ is the mach number, and $\gamma$ is the isentropic coefficient of air. The air present in the cavity is heated by the discharge but during the expulsion it is cooled by the cavity. As it is impossible to measure the temperature during the expulsion phase, the temperature is assumed to be equal to $293 \mathrm{~K}$, such that the isentropic coefficient is equal to 1.4. The expression for the velocity is then:

$$
V_{\max }=\sqrt{\frac{2 \cdot \gamma \cdot R \cdot T}{\gamma-1}\left(\left(\frac{P_{t \max }}{P_{a}}\right)^{\frac{\gamma-1}{\gamma}}-1\right)}
$$

The curves resulting from this calculation are shown in Figure 17.

\subsection{Comparison between schlieren velocity measurements and the velocity calculation}

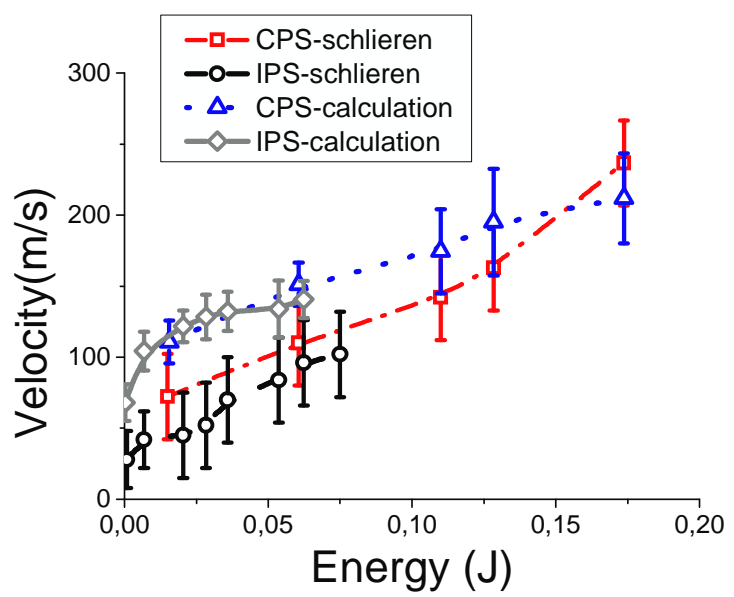

Figure 17. Computed and measured maximum jet velocities as a function of the discharge energy 
With both power supplies, the velocity increases as a function of the energy dissipated in the discharge. The PSJ jet is produced by heating of the air in the cavity, involving an increase in the static pressure. The pressure gradient between the heated air in the cavity and the external air leads to the creation of the jet. The stronger the pressure gradient is, the higher the jet Mach number is. The jet produced by the PSJ actuator supplied by the IPS has a lower velocity than the jet produced by the PSJ actuator supplied by the CPS (as seen in the schlieren measurement). We have observed than the cap temperature is higher for IPS than for the CPS, the thermal loss is then more important for the IPS. The part of the energy involved in the gas expulsion is lower. IPS dissipates too much energy in heating PSJ the ceramic cavity and enough in the heating of the gas present in the cavity before breakdown, because of the expulsion begins when the discharge is not finished as seen on Figure 13. Moreover, as seen in Figure 17, there are differences between the velocities determined from the schlieren images and those calculated from pressure measurements, indicating that the modeled velocity, based on these measurements, is not perfect. This outcome shows that the assumption of an isentropic flow of the jet is incorrect. The hypothesis of an isentropic flow does not take heat losses into account, which lead to heating of the cavity by the discharge. The consequence of the heat loss is an increase in the temperature of the cavity, and thus an increase in the gas temperature.

Moreover, it can be seen that the differences between the schlieren image velocities and the pressure model velocities are greater for the inductive than for the CPS. This suggests that the heat loss or temperatures are greater when the jet is created by the IPS. This explains why the temperature of the cap is higher for the IPS than the CPS. This conclusion is supported by the fact that the breakdown voltage obtained with the IPS is lower than that obtained with the CPS, meaning that the jet created by the IPS is hotter than that created by the CPS.

\section{Conclusions}

The influence of the two power supplies on the plasma is very different: 
- In the case of the IPS, the discharge is equivalent to a constant voltage. The current is thus controlled by the transformer, and depends on the energy accumulated in its magnetizing inductance. Consequently, the discharge duration increases with the energy dissipated on the plasma. Moreover, the discharge is relatively long, such that the energy is dissipated relatively slowly.

- The CPS creates a pulsed discharge, which is controlled by a capacitor. This discharge resembles a spark discharge and behaves like a small and constant resistance from the energetic point of view, such that the energy is dissipated very quickly.

The energy dissipation rate influences the warming of the gas present in the cavity, and consequently the gas expulsion time. This time interval is longer for the IPS, and is influenced by the energy dissipated in the cavity.

Moreover, the front jet velocity is influenced by the energy dissipation rate in the cavity, as shown by the velocities measured with the schlieren bench, which are lower with the IPS. The energy dissipation rate also influences the temperature of the gas, and thus the heat loss, as can be seen from the breakdown voltage and the velocity calculation. Indeed, the breakdown voltage decreases with the temperature of the gas before breakdown, and is lower for the IPS than for the CPS. Furthermore, the differences between the velocities derived from the pressure model, and those determined from the schlieren images, also indicate that the IPS heats the gas and the cavity of the PSJ actuator more strongly than does the CPS.

The energy dissipation rate is a very important parameter, because it influences the velocity and heating of the PSJ actuator. In order to increase the efficiency of the PSJ actuator, it is very important to diminish the energy dissipation rate in the discharge. The CPS thus produces a more powerful jet (higher velocity, shorter expulsion time), and probably heats the gas and the cavity less significantly than the IPS. In order to further increase the velocity of the jet, and decrease the heat losses, it would be beneficial to develop a ns-pulse power supply, able to supply $1 \mathrm{~mJ}$ in an interval of a few nanoseconds $[37,38]$.

\section{Aknowledgments}


The research leading to these results has received funding from the European Community's Seventh

Framework Programme FP7/2007-2013 under the PLASMAERO grant agreement n²34201.

\section{REFERENCE:}

1. Smith BL, Glezer A. 1998 The formation and evolution of synthetic jets Phys. Fluids. 10 2281

2. Mane P, Mossi K, Bryant R. 2008 Experimental design and analysis for piezoelectric circular actuators in flow control applications Smart Mater. Struct. 17015013.

3. Wiltse JM, Glezer A. 1998 Direct excitation of small-scale motions in free shear flows Phys. Fluids.10 2026.

4. Roth JR. 2003 Aerodynamic flow acceleration using paraelectric and peristaltic electrohydrodynamic effects of a One Atmosphere Uniform Glow Discharge Plasma Phys. Plasmas. 102117.

5. Moreau E. 2007 Airflow control by non-thermal plasma actuators J. Phys. D: Appl. Phys. 40 605-636.

6. Hyun KT, Chun CH. 2003 The wake flow control behind a circular cylinder using ion wind Exp Fluids 35 541-552.

7. Corke TC, Post ML, Orlov DM. 2008 Single dielectric barrier discharge plasma enhanced aerodynamics: physics, modeling and applications Exp Fluids. 46 1-26.

8. Boeuf JP, Pitchford LC. 2005 Electrohydrodynamic force and aerodynamic flow acceleration in surface dielectric barrier discharge J. Appl. Phys. 97103307.

9. Benard N, Mizuno A, Moreau E. 2009 A large-scale multiple dielectric barrier discharge actuator based on an innovative three-electrode design J. Phys. D: Appl. Phys. 42 235204.

10. Benard N, Jolibois J, Moreau E, Sosa R, Artana G, Touchard G. 2008 Aerodynamic plasma actuators: A directional micro-jet device. Thin Solid Films. 516 6660-6667.

11. Cybyk BZ, Wilkerson JT, Grossman KR. 2004 Performance Characteristics of the sparkjet flow control actuator 2nd AIAA Flow Control Conference (Portland) p. 20042131.

12. Cybyk BZ, Grossman KR, Wilkerson JT. 2005 Single-Pulse Performance of the sparkjet flox control actuator 43rd AIAA Aerospace Sciences Meeting and Exhibit (Reno) p. 2005-401.

13. Ko H, Haack S, Land H, Cybyk B, Katz J, Kim H. 2010 Analysis of flow distribution from high-speed flow actuator using particle image velocimetry and digital speckle tomography Flow. Meas. Instrum. 21 443-453.

14. Narayanaswamy V, Raja LL, Clemens NT. 2010 Characterization of a High-Frequency Pulsed-Plasma Jet Actuator for Supersonic Flow Control AIAA Journal. 48 297-305.

15. Caruana D, Barricau P, Hardy P, Cambronne J, Belinger A. 2009 The "Plasma Synthetic Jet" Actuator: Aero-thermodynamic Characterization and first Flow Control Applications 47th AIAA Aerospace Sciences Meeting. (Orlando) p. 2009-1307.

16. Mildren R, Carman R. 2001 Enhanced performance of a dielectric barrier discharge lamp using short-pulsed excitation. J. Phys. D: Appl. Phys. 34 L1-L6.

17. Mildren R, Carman R, Falconer I. 2002 Visible and VUV emission from a xenon dielectric barrier discharge using pulsed and sinusoidal voltage excitation waveforms Plasma Science, IEEE Transactions on. 30 192-193. 
18. Liu S, Neiger M. 2003 Double discharges in unipolar-pulsed dielectric barrier discharge xenon excimer lamps J. Phys. D: Appl. Phys. 36 1565-1572.

19. Williamson JM, Trump DD, Bletzinger P, Ganguly BN. 2006 Comparison of high-voltage ac and pulsed operation of a surface dielectric barrier discharge. J. Phys. D: Appl. Phys. 39 4400-4406.

20. Koudriavtsev O, Shengpei Wang, Konishi Y, Nakaoka M. 2002 A novel pulse-densitymodulated high-frequency inverter for silent-discharge-type ozonizer IEEE Transactions on Industry Applications, 38 369-378

21. Ordiz C, Alonso J, Costa M, Ribas J, Calleja A. 2008 Development of a high-voltage closed-loop power supply for ozone generation APEC 2008. Twenty-Third Annual IEEE. p. 1861-1867

22. Jolibois J, Moreau E. 2009 Enhancement of the Electromechanical Performances of a Single Dielectric Barrier Discharge Actuator Dielectrics and Electrical Insulation, IEEE Transactions on. 16 758-767.

23. Balcon N, Benard N, Lagmich Y, Boeuf J, Touchard G, Moreau E. 2009 Positive and negative sawtooth signals applied to a DBD plasma actuator - influence on the electric wind J. Electrostat. 67 140-145.

24. Benard N, Moreau E. 2010 Capabilities of the dielectric barrier discharge plasma actuator for multi-frequency excitations J. Phys. D: Appl. Phys. 43145201.

25. Redondo LM, Silva JF. Flyback Versus Forward Switching Power Supply Topologies For Unipolar Pulsed-Power Applications. IEEE Transactions on Plasma Science. 2009 janv;37(1):171-178.

26. Hnatiuc B, Hnatiuc E, Pellerin S, Chapelle J. Experimental analysis of a double-spark ignition system. Czech J Phys. 2006 août;56(8):851-867.

27. Zaepffel C, Hong D, Bauchire JM. Experimental study of an electrical discharge used in reactive media ignition. Journal of Physics D (Applied Physics). 2007;40(4):1052-1058.

28. Granda-Gutiérrez E, López-Callejas R, Peña-Eguiluz R, Benítez-Read J, Pacheco-Sotelo J, Valencia A. R, et al. 2007 Modelling and optimization of a low-pressure DC glow discharge in stable regime Surf. Coat. Technol. 201 5454-5457.

29. Takaki K, Taguchi D, Fujiwara T. 2001 Voltage-current characteristics of high-current glow discharges Appl. Phys. Lett. 782646.

30. Staack D, Farouk B, Gutsol A, Fridman A. 2005 Characterization of a dc atmospheric pressure normal glow discharge Plasma Sources Sci. Technol. 14 700-711.

31. Greason W, Kucerovsky Z, Bulach S, Flatley M. 1997 Investigation of the optical and electrical characteristics of a spark gap IEEE Transactions on Industry Applications,. 33 1519-1526.

32. Greason W. 1999 Methodology to study the resistance of spark discharges IEEE Transactions on Industry Applications, 35 359-365.

33. Randeberg E, Olsen W, Eckhoff RK. 2006 A new method for generation of synchronised capacitive sparks of low energy J. Electrostat. 64 263-272.

34. Papadopoulos AD, Serafetinides AA. 1991 Investigation of the electrical characteristics of charge transfer circuits used in gas laser excitation J. Phys. D: Appl. Phys. 24 19171924.

35. Persephonis P, Vlachos K, Georgiades C, Parthenios J. 1992 The inductance of the discharge in a spark gap J. Appl. Phys. 71 4755-4762.

36. Persephonis P, Giannetas V, Ioannou A, Parthenios J, Georgiades C. 1996 The time evolution of the resistances and inductances of the discharges in a pulsed gas laser through its current waveforms., IEEE Transactions on Plasma Science. 24 1208-1214. 
37. Pai DZ, Lacoste DA, Laux CO. Transitions between corona, glow, and spark regimes of nanosecond repetitively pulsed discharges in air at atmospheric pressure. J. Appl. Phys. 2010;107(9):093303.

38. Pai DZ, Lacoste DA, Laux CO. Nanosecond repetitively pulsed discharges in air at atmospheric pressure-the spark regime. Plasma Sources Sci. Technol. 2010 déc;19(6):065015. 


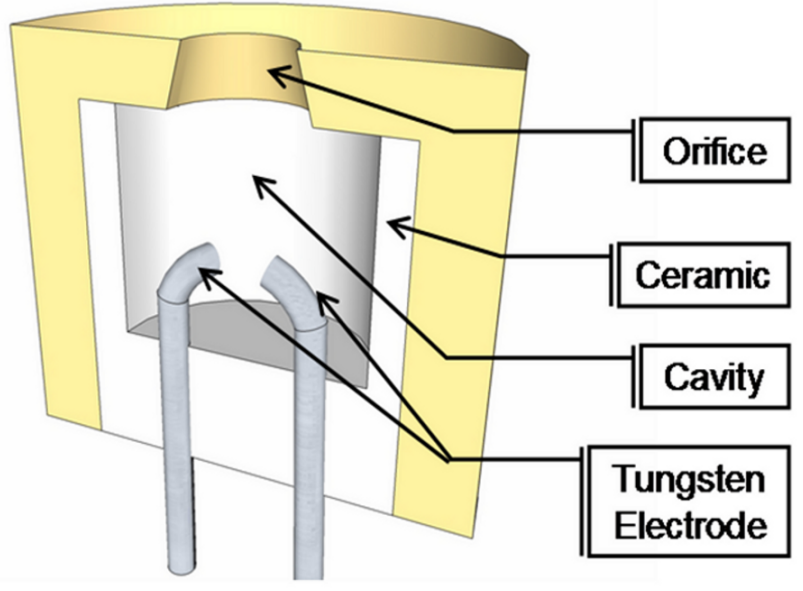

$$
\text { (a) }
$$

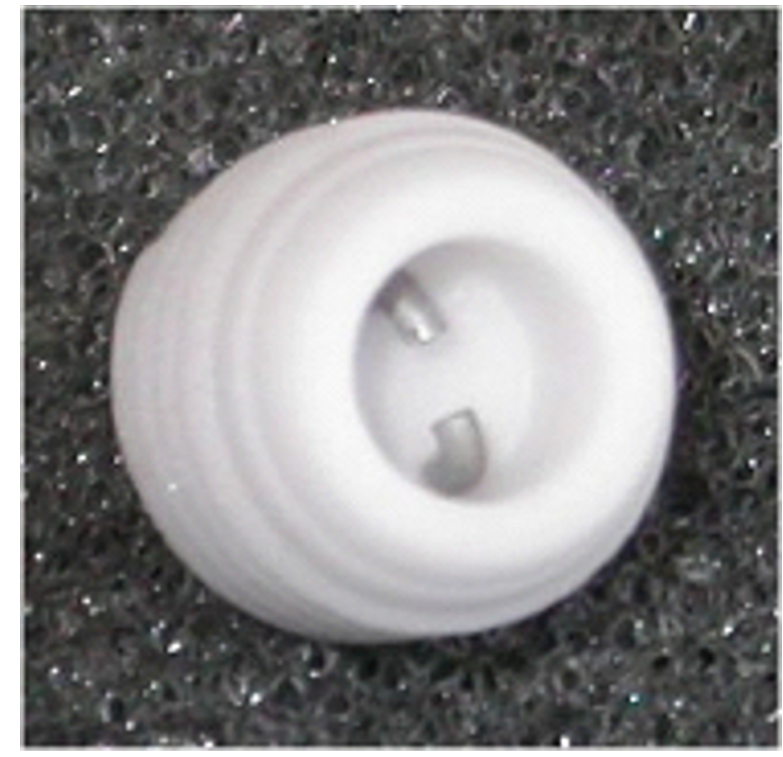

( b )

Figure 1 (Belinger-PSJ-correction/figure1.tif) 


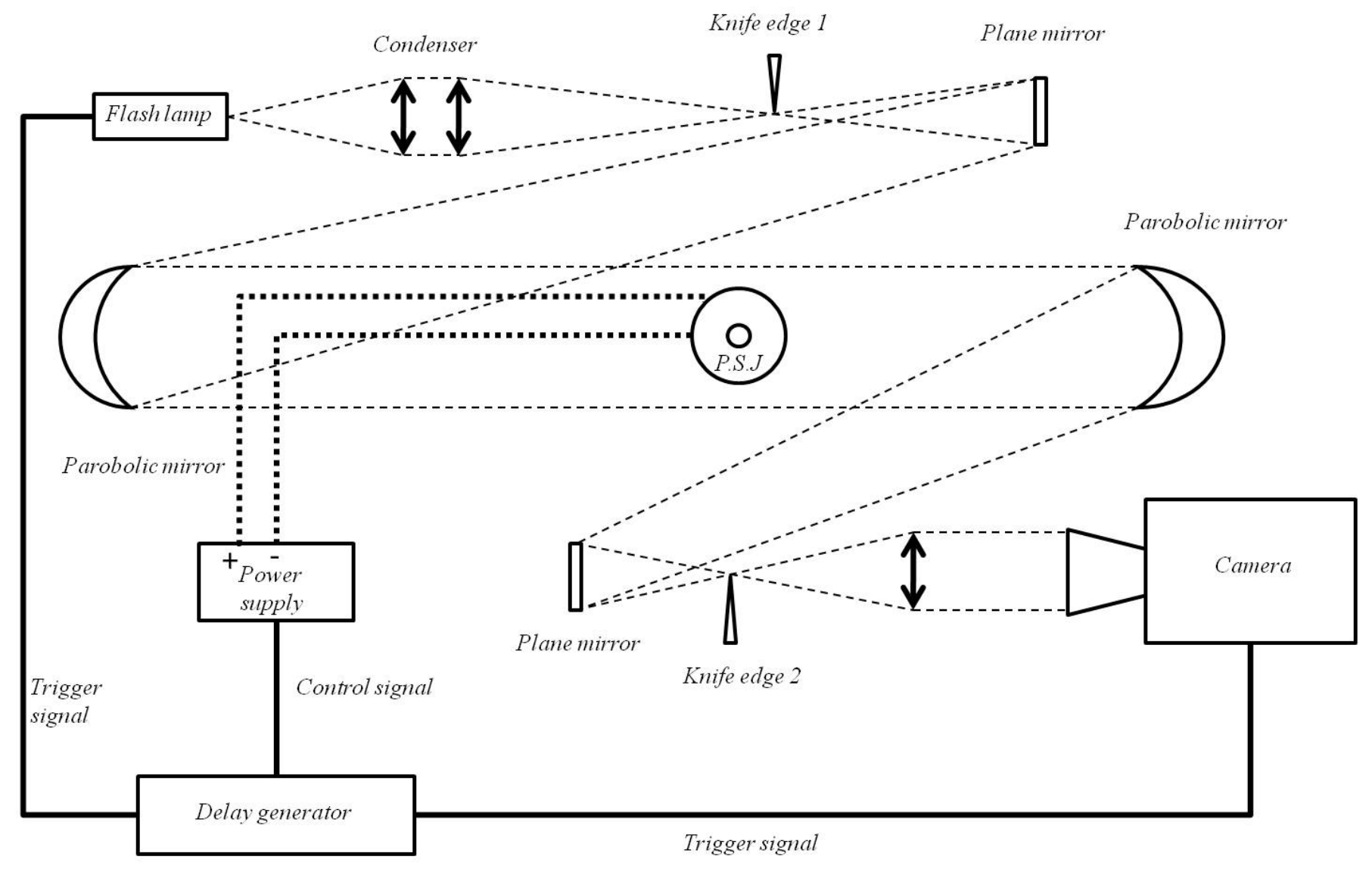

Figure 2 (Belinger-PSJ-correction/figure2.jpg) 


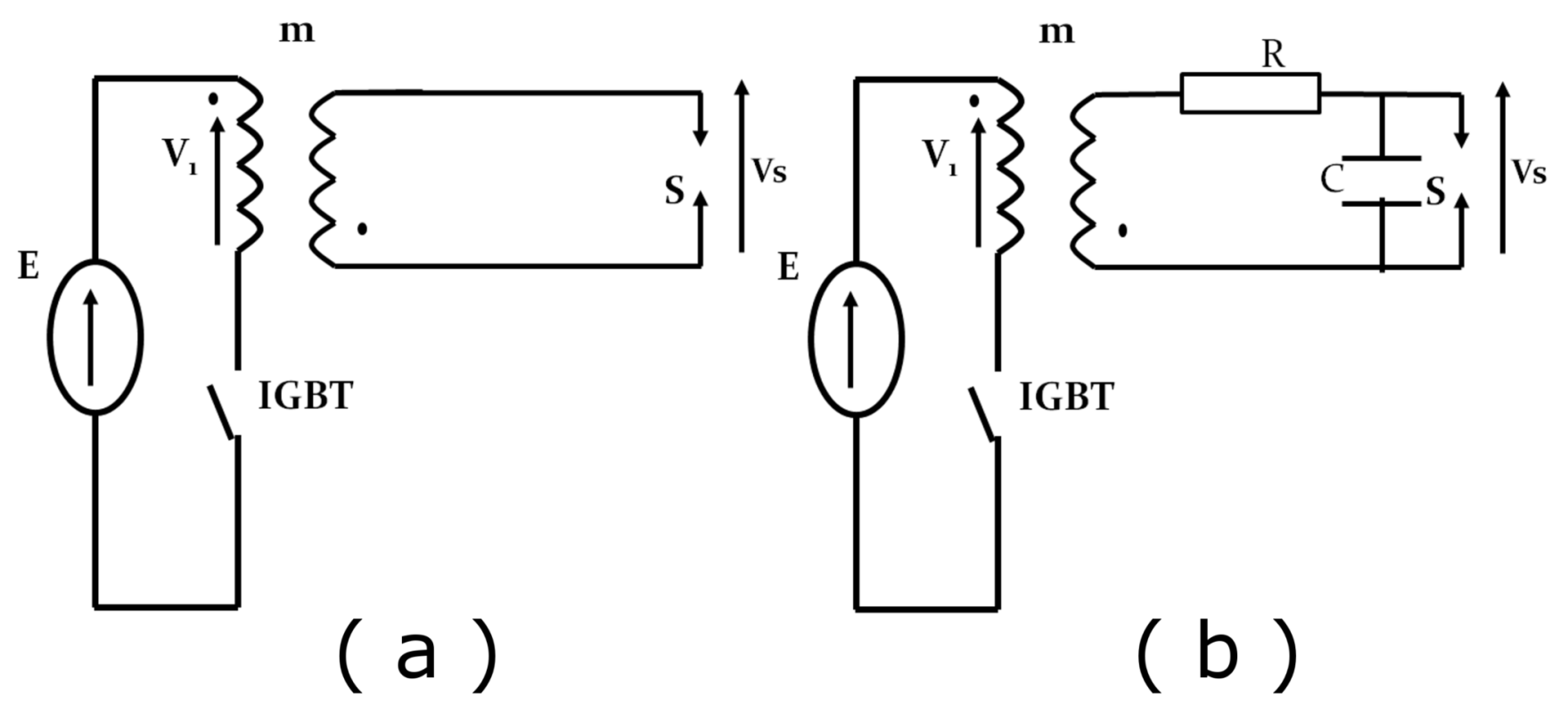

Figure 3 (Belinger-PSJ-correction/figure3.tif) 


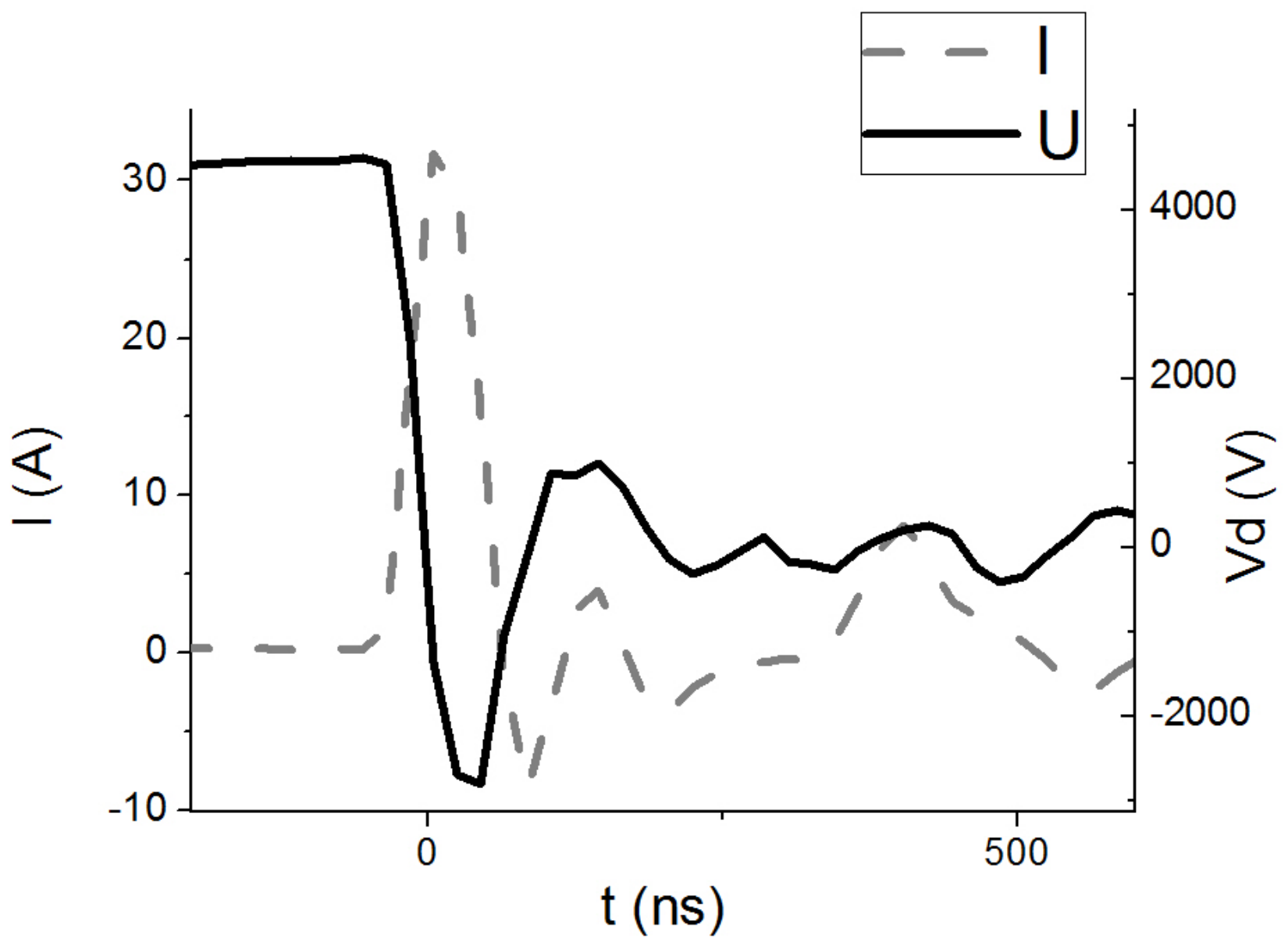

Figure 4 (Belinger-PSJ-correction/figure4.jpg) 


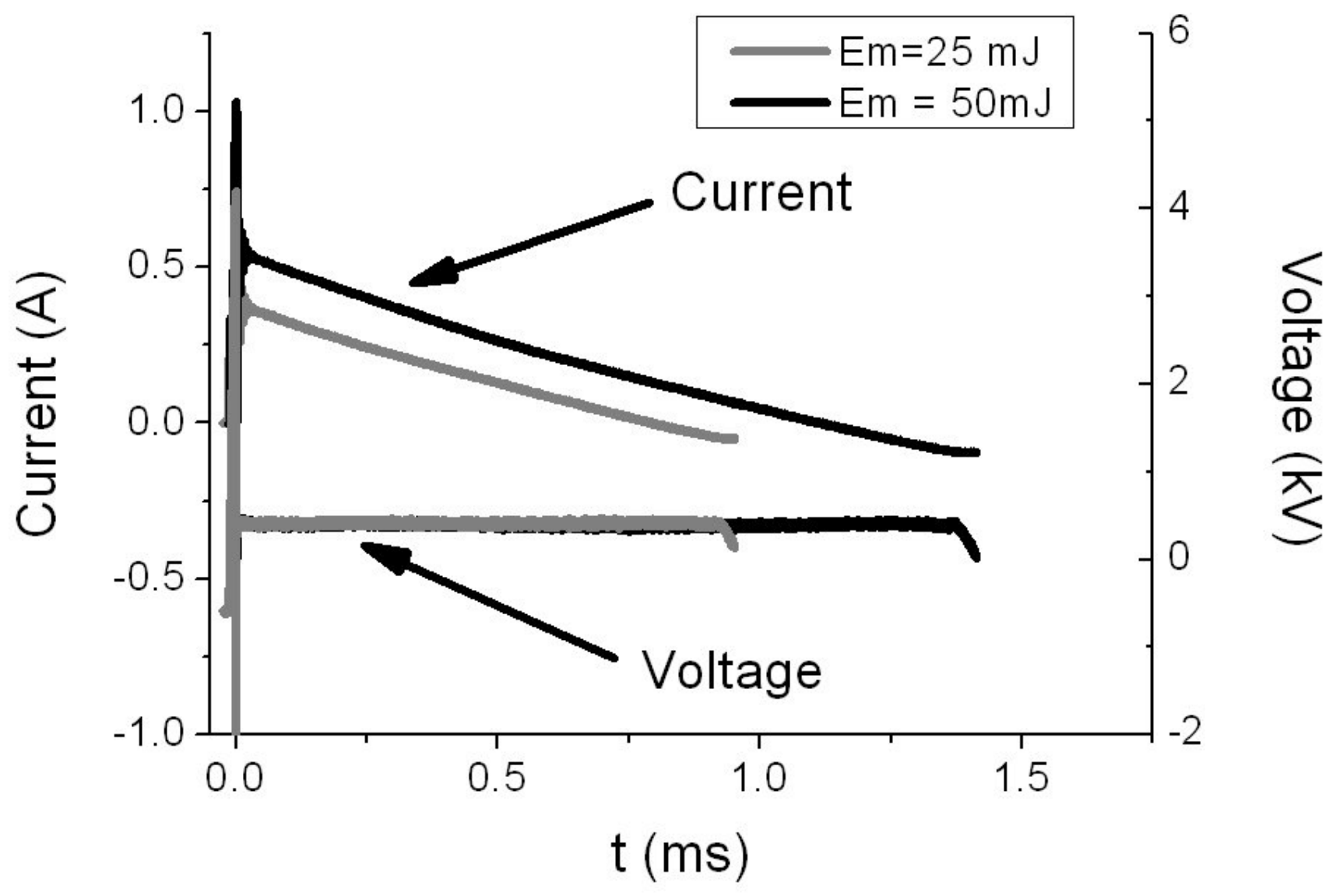

Figure 5 (Belinger-PSJ-correction/figure5.jpg) 


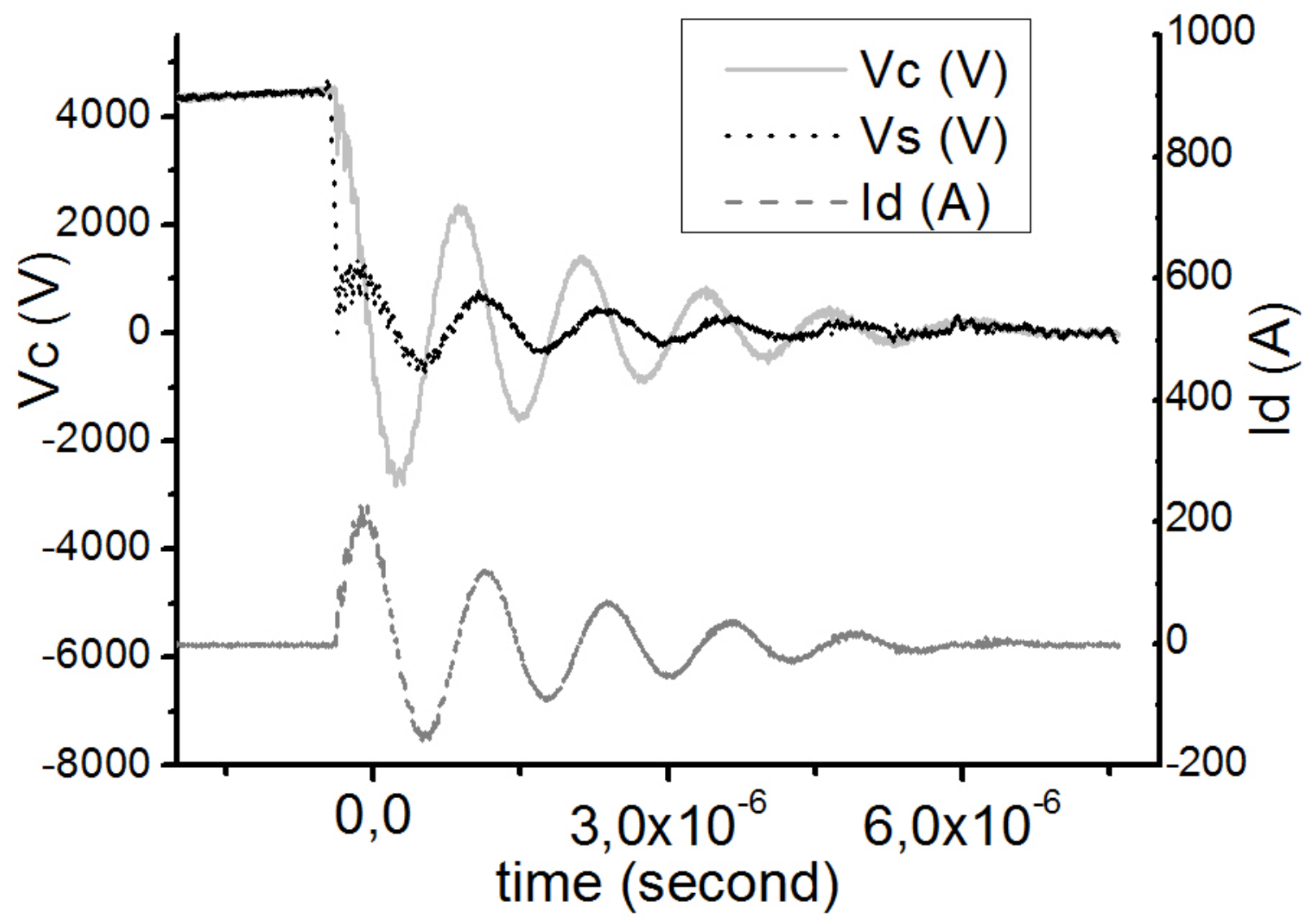

Figure 6 (Belinger-PSJ-correction/figure6.jpg) 


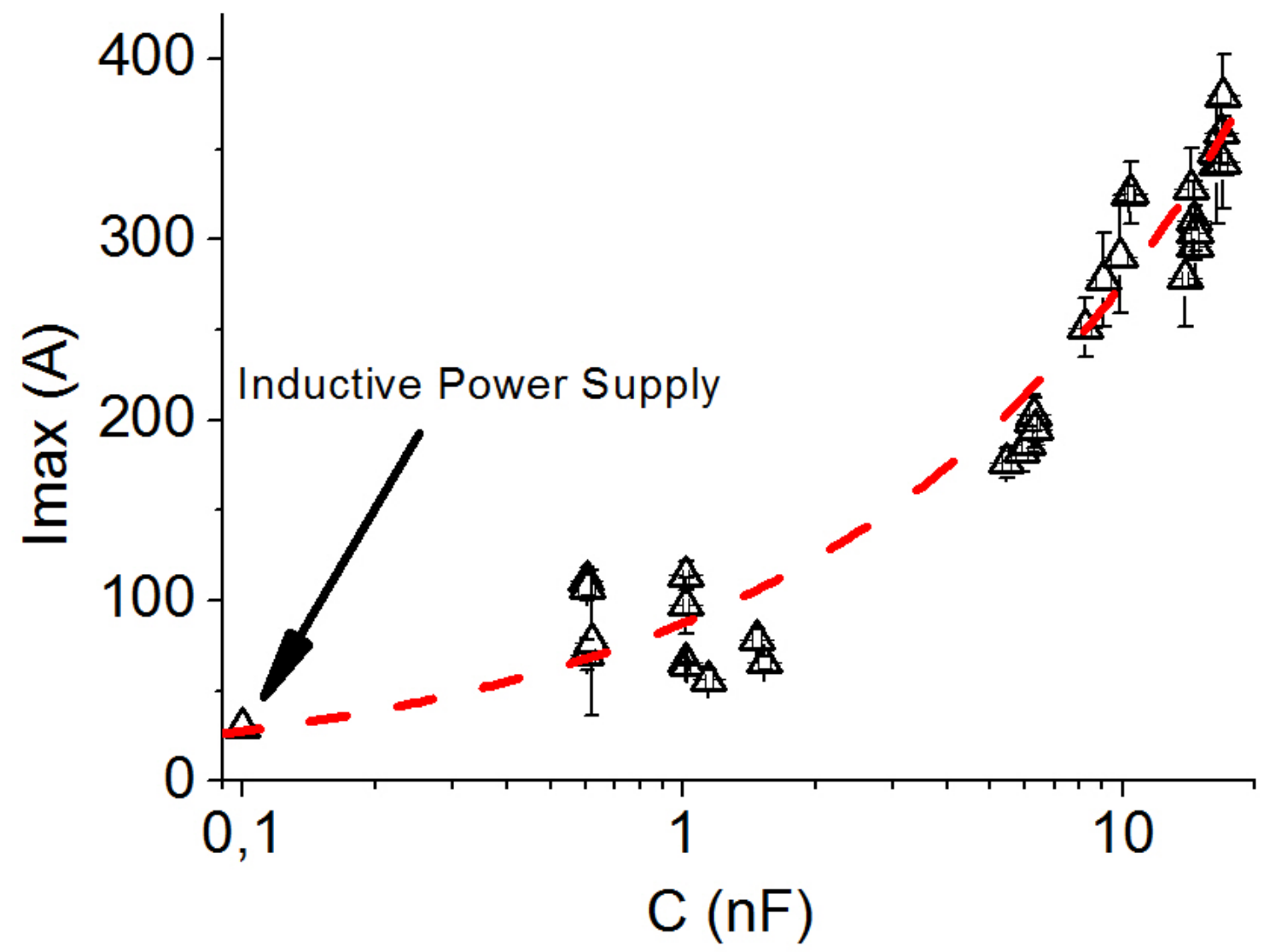

Figure 7 (Belinger-PSJ-correction/Figure7.jpg) 


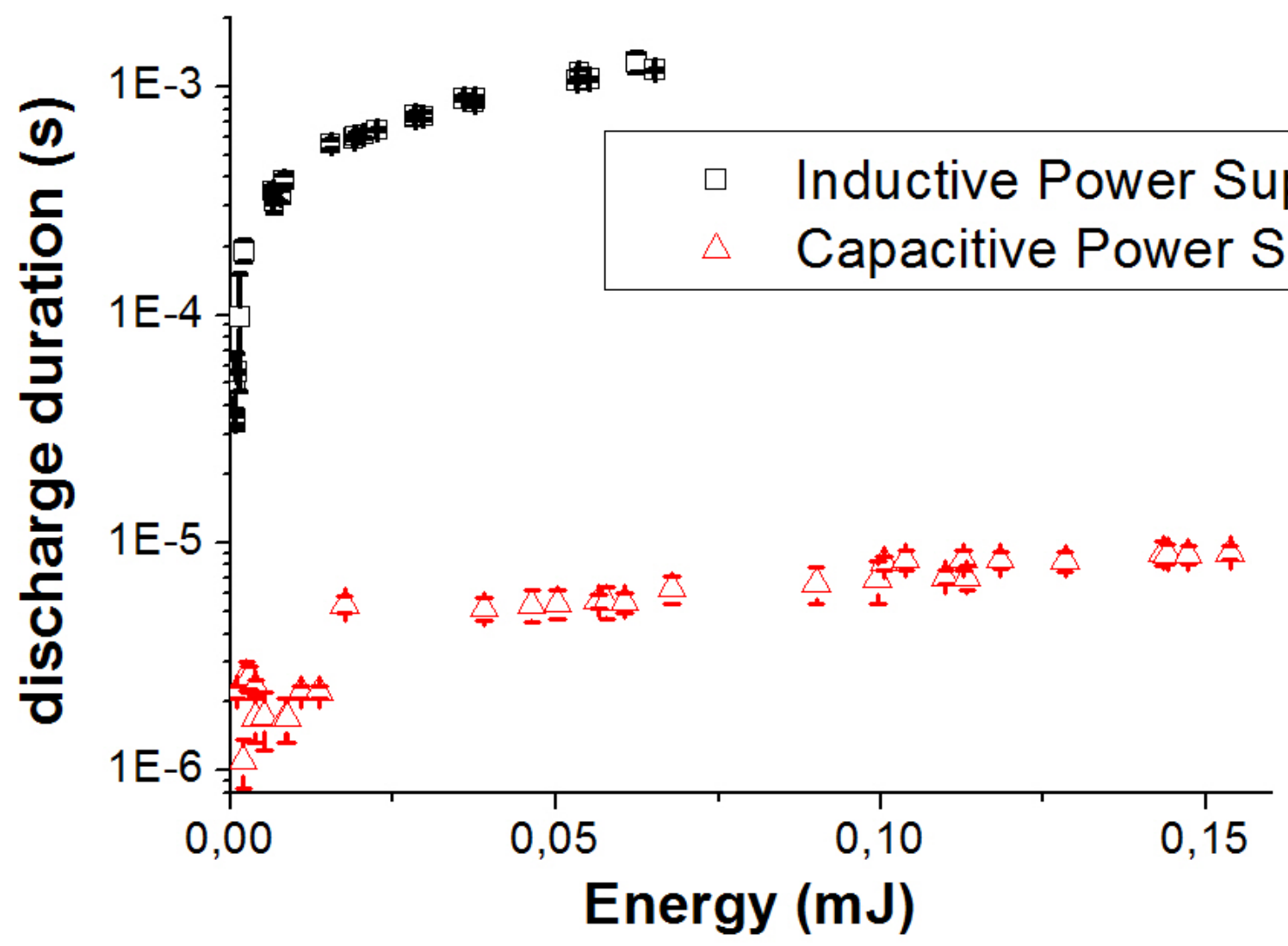

Figure 8 (Belinger-PSJ-correction/figure8.jpg) 


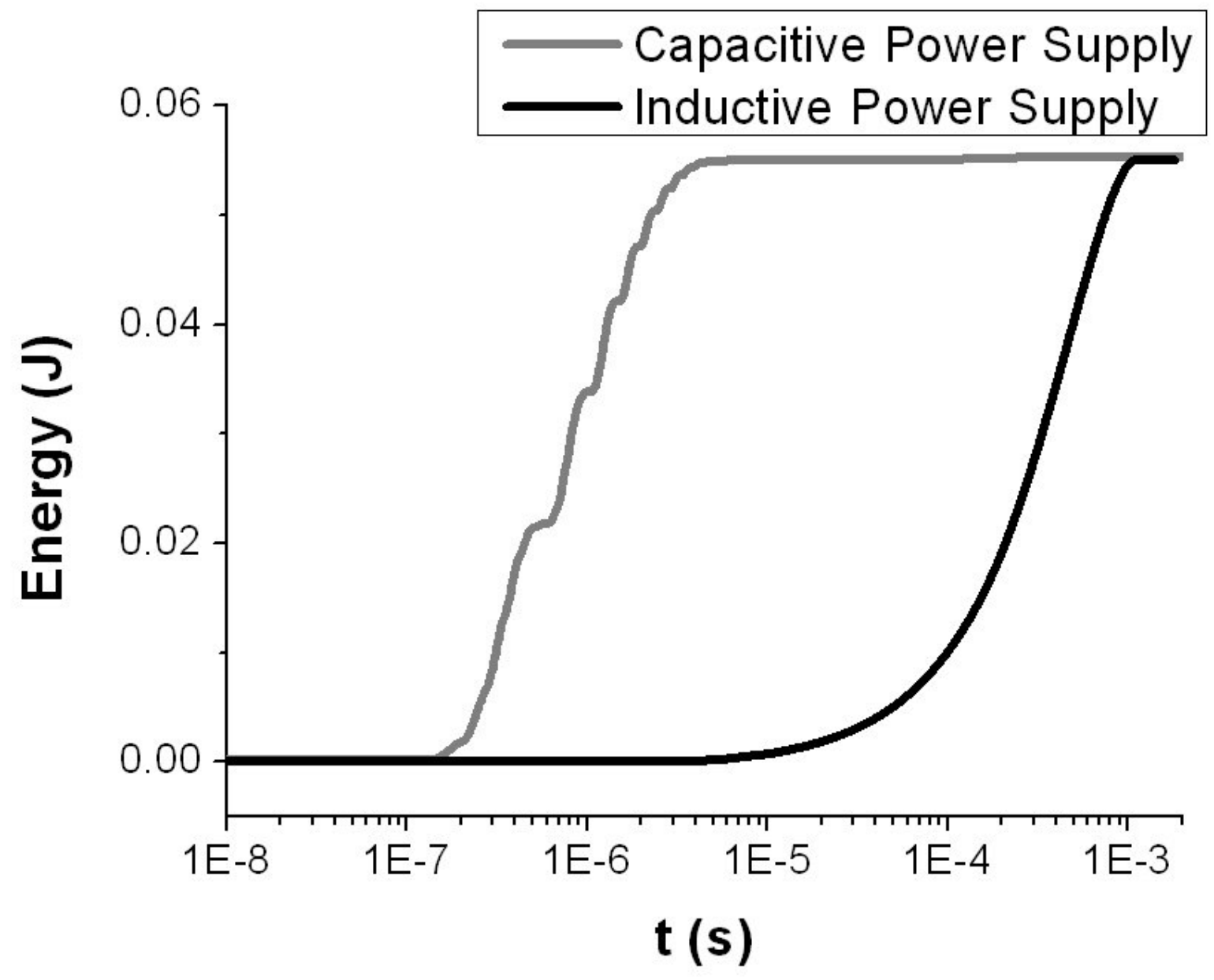

Figure 9 (Belinger-PSJ-correction/figure9.jpg) 


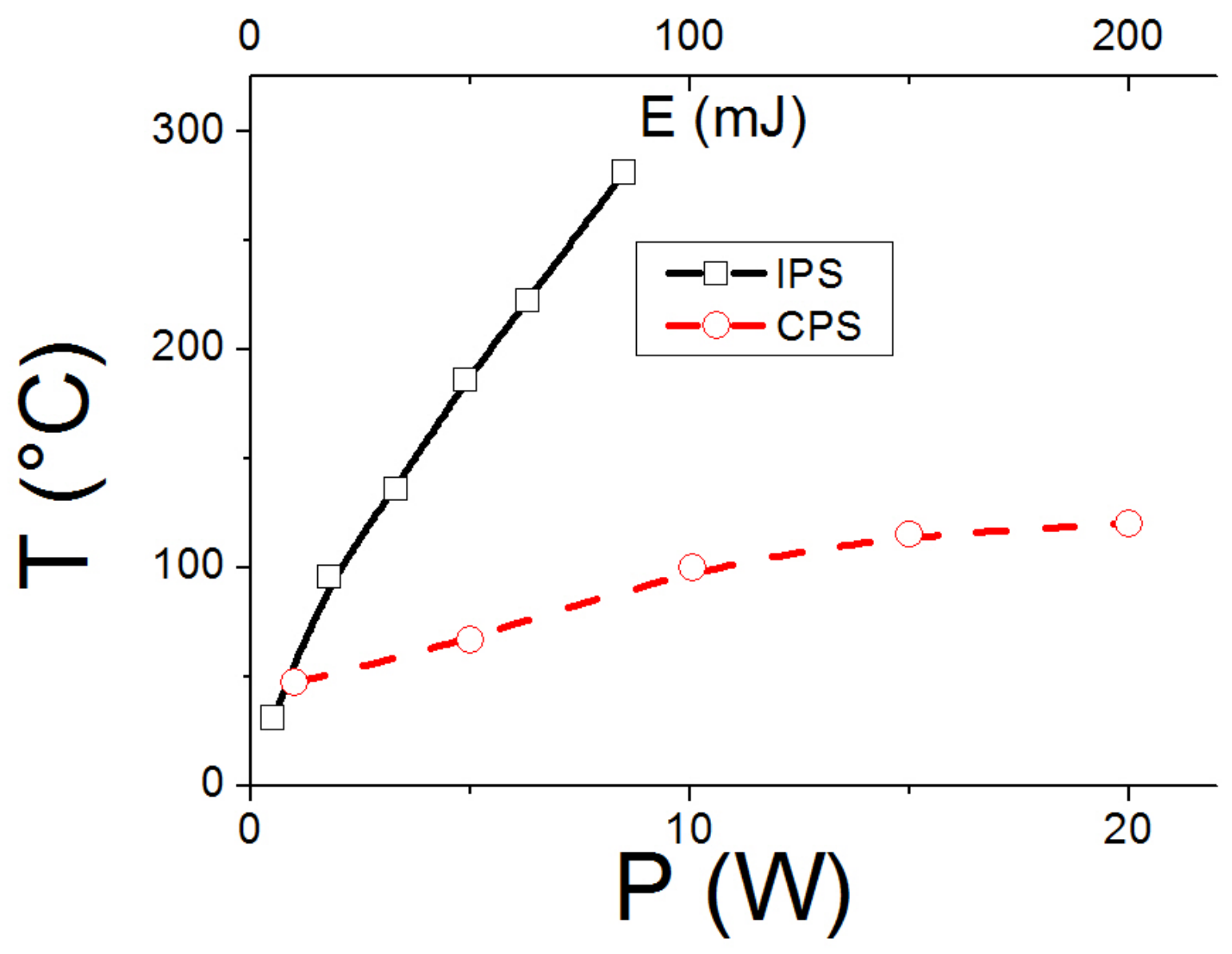

Figure 10 (Belinger-PSJ-correction/figure10.jpg) 


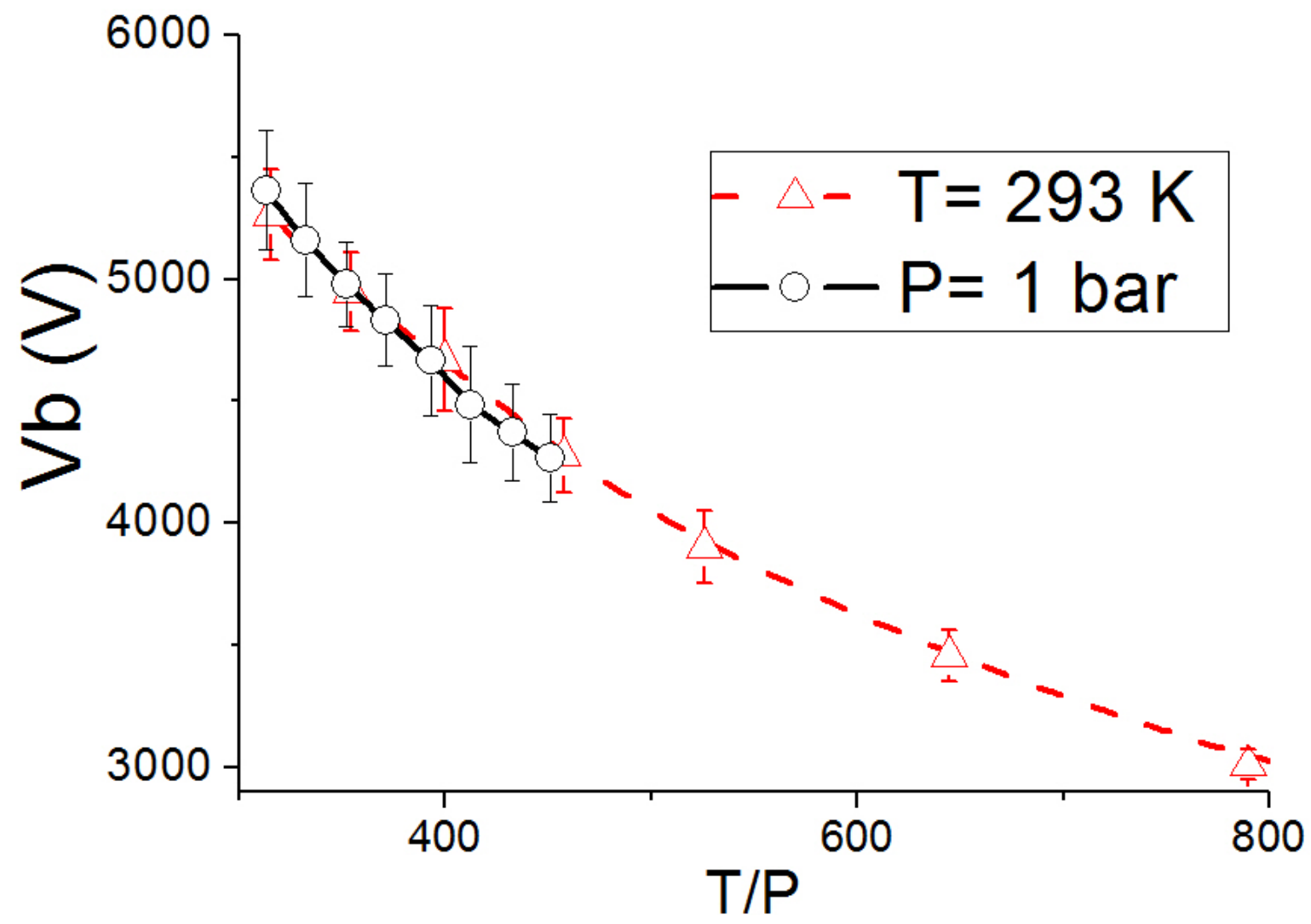

Figure 11 (Belinger-PSJ-correction/Figure11.jpg) 


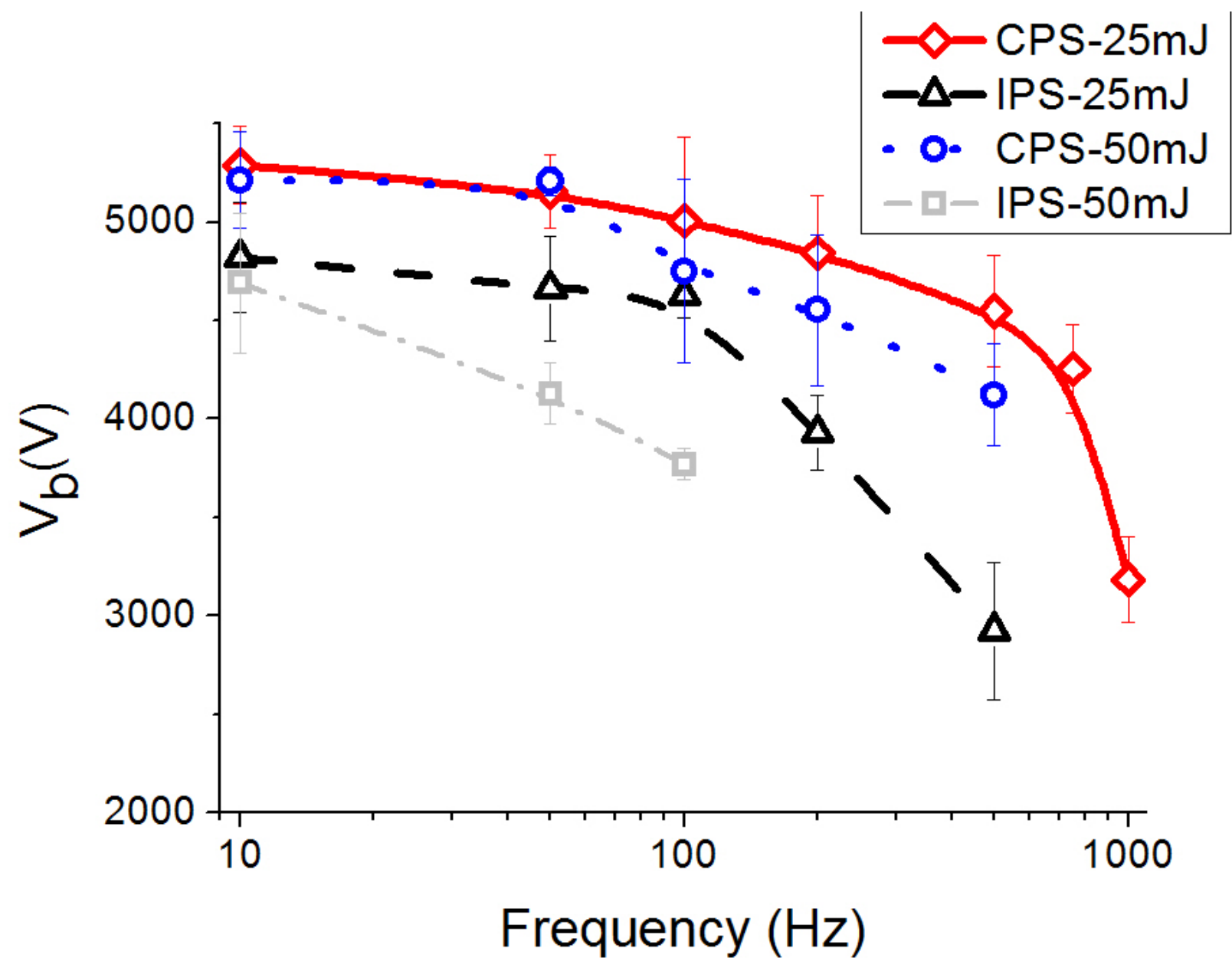

Figure 12 (Belinger-PSJ-correction/Figure12.jpg) 


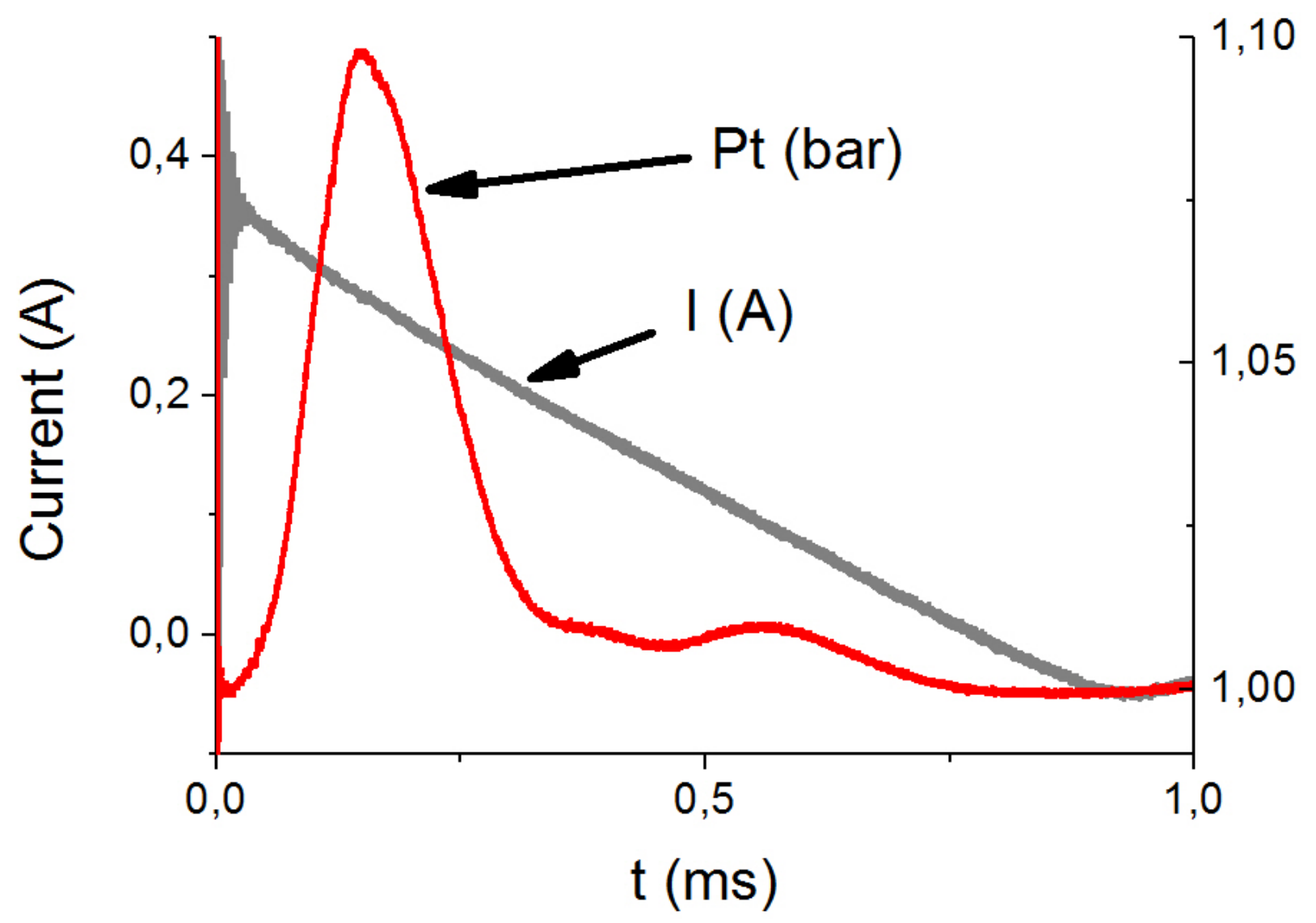

Figure 13 (Belinger-PSJ-correction/figure13.jpg) 


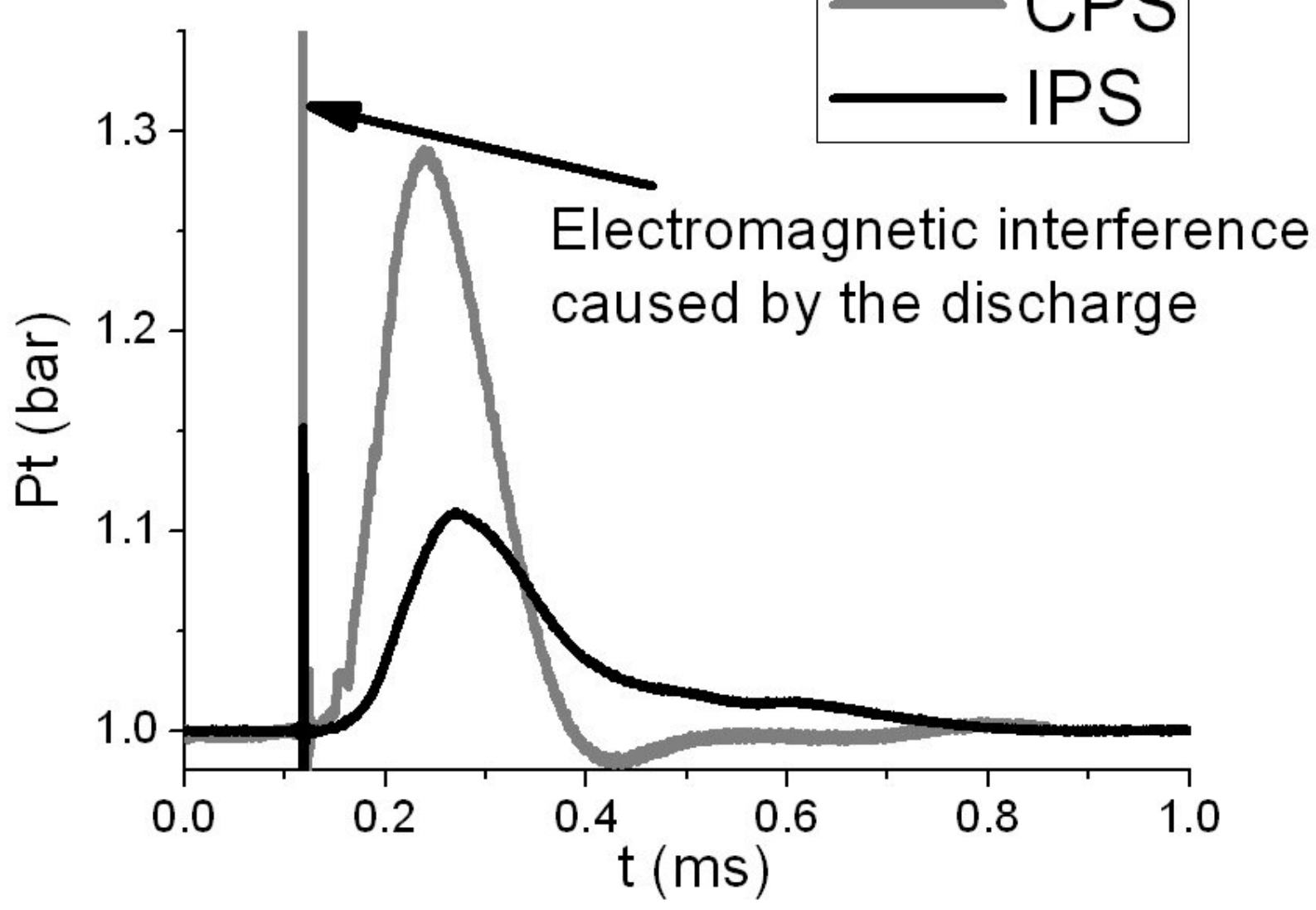

Figure 14 (Belinger-PSJ-correction/figure14.jpg) 


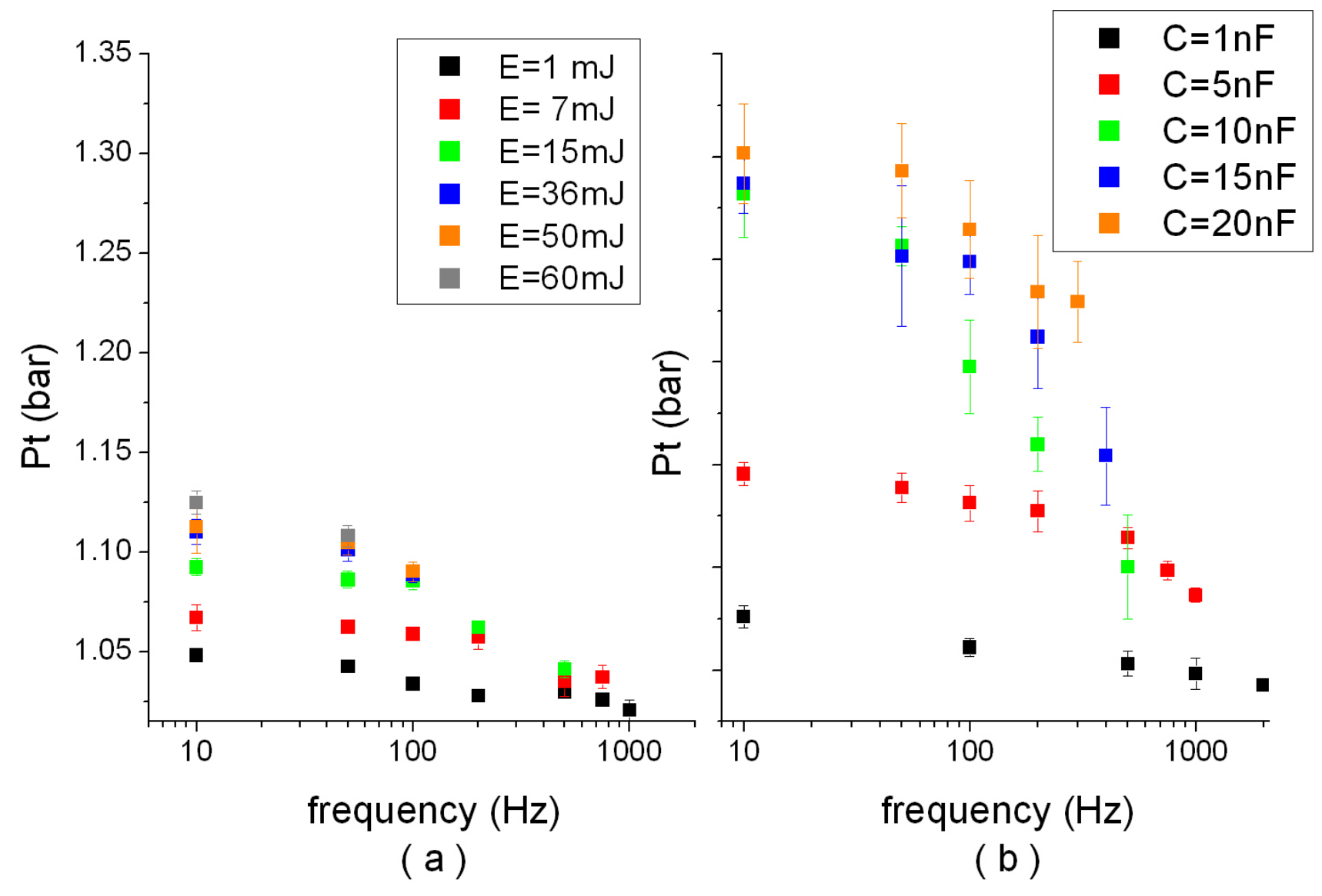

Figure 15 (Belinger-PSJ-correction/figure15.jpg) 


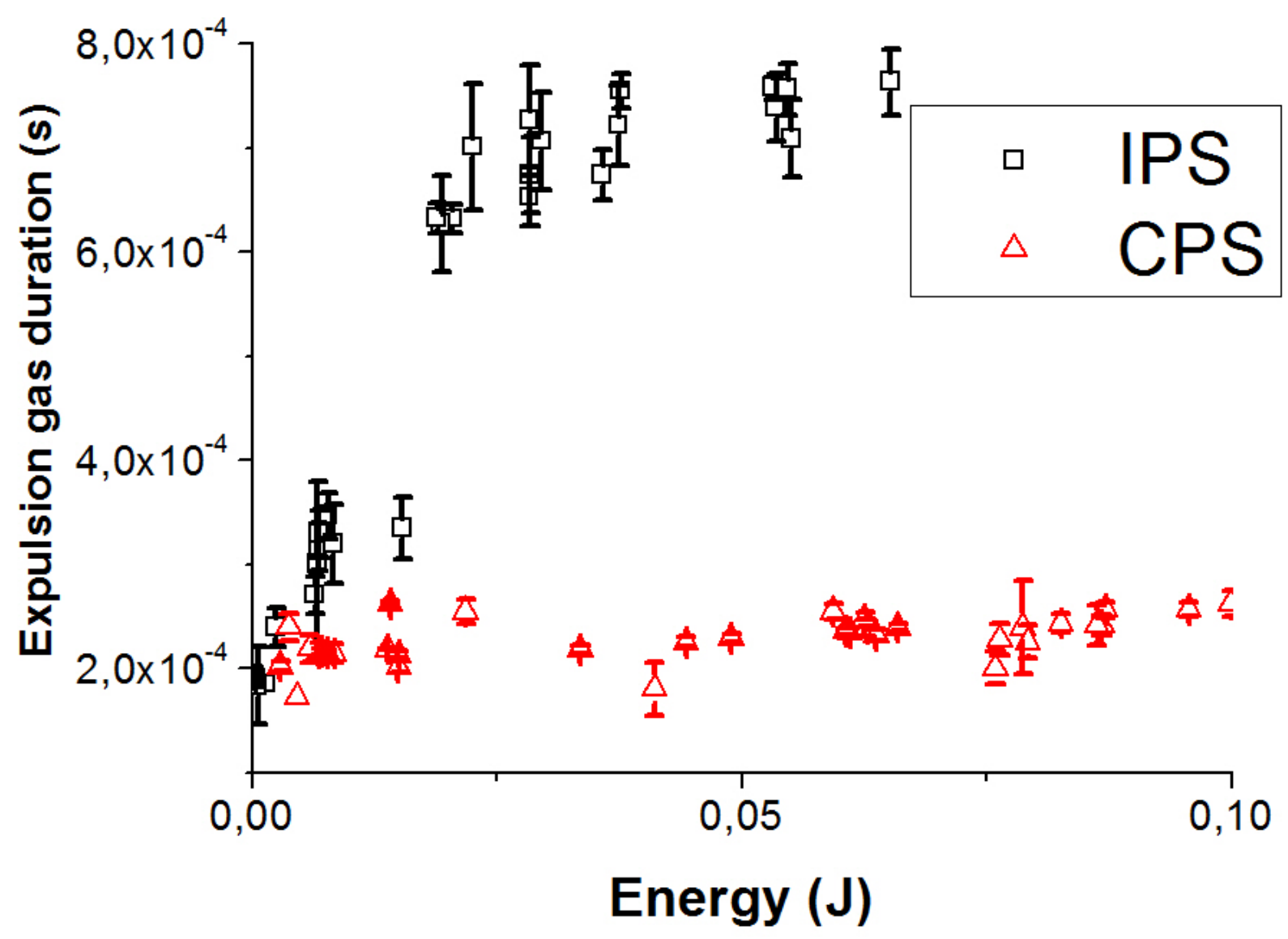

Figure 16 (Belinger-PSJ-correction/figure16.jpg) 


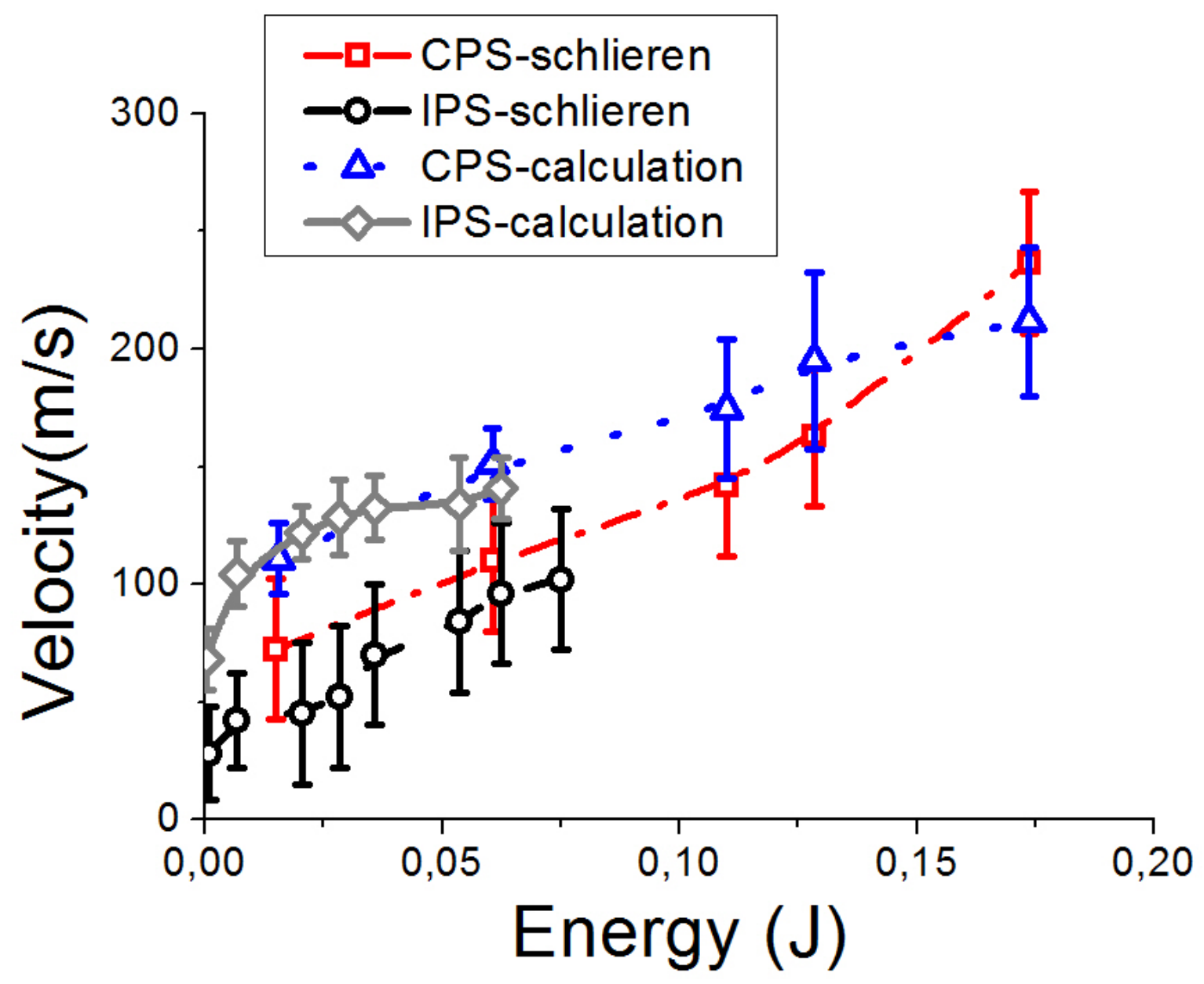

Figure 17 (Belinger-PSJ-correction/figure17.jpg) 